Linköping Studies in Science and Technology

Thesis No. 1748

\title{
Titanium oxide nanoparticle production using high power pulsed plasmas
}

\author{
Rickard Gunnarsson
}

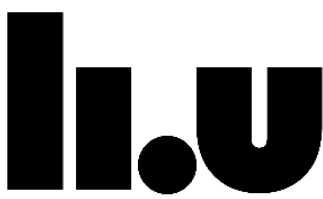

LINKÖPING UNIVERSITY

Plasma \& Coatings Physics Division

Department of Physics Chemistry and Biology

Linköping University, SE-581 83 Linköping, Sweden

Linköping 2016 
(C) Rickard Gunnarsson, 2016

Printed in Sweden by LiU-Tryck

ISSN 0280-7971

ISBN 978-91-7685-766-3 


\section{Abstract}

This thesis covers fundamental aspects of process control when growing titanium oxide nanoparticles in a reactive sputtering process. It covers the influence of oxygen containing gas on the oxidation state of the cathode from which the growth material is ejected, as well as its influence on the particles oxidation state and their nucleation. It was found that a low degree of reactive gases was necessary for nanoparticles of titanium to nucleate. When the oxygen gas was slightly increased, the nanoparticle yield and particle oxygen content increased. A further increase caused a decrease in particle yield which was attributed to a slight oxidation of the cathode. By varying the oxygen flow to the process, it was possible to control the oxygen content of the nanoparticles without fully oxidizing the cathode. Because oxygen containing gases such as residual water vapour has a profound influence on nanoparticle yield and composition, the deposition source was re-engineered to allow for cleaner and thus more stable synthesis conditions.

The size of the nanoparticles has been controlled by two means. The first is to change electrical potentials around the growth zone, which allows for nanoparticle size control in the order of $25-75 \mathrm{~nm}$. This size control does not influence the oxygen content of the nanoparticles. The second means of size control investigated was by increasing the pressure. By doing this, the particle size can be increased from $50-250 \mathrm{~nm}$, however the oxygen content also increases with pressure. Different particle morphologies were found by changing the pressure. At low pressures, mostly spherical particles with weak facets were produced. As the pressure increased, the particles got a cubic shape. At higher pressures the cubic particles started to get a fractured surface. At the highest pressure investigated, the fractured surface became poly-crystalline, giving a cauliflower shaped morphology. 


\section{Preface}

This thesis is part of my PhD studies in the Plasma \& Coatings Physics division of the department of Physics, Chemistry and Biology at Linköping University. The goal of my research is to study and understand the nucleation and growth of titanium nanoparticles synthesized in a novel pulsed plasma discharge. This research is financially supported by the Knut and Alice Wallenberg foundation. 


\section{List of appended papers}

Paper 1 Synthesis of titanium-oxide nanoparticles with size and stoichiometry control

Rickard Gunnarsson, Ulf Helmersson, Iris Pilch

Journal of Nanoparticle Research 17, 1-11 (2015)

Paper 2 The influence of pressure and gas flow on size and morphology of titanium oxide nanoparticles synthesized by hollow cathode sputtering

Rickard Gunnarsson, Iris Pilch, Robert Boyd, Nils Brenning, Ulf Helmersson

Submitted

The author's contribution to the appended papers

In paper 1 I assembled the experimental setup, performed all of the experiments, characterized the nanoparticles with SEM and XRD and wrote a major part of the paper.

In paper 2 I designed and constructed the new experimental setup, participated in planning the experiments, preformed all of the experiments, characterized the particles with SEM, prepared the first draft and wrote a major part of the paper 


\section{Related Publications not included in this thesis}

Highly reflective rear surface passivation design for ultra-thin $\mathrm{Cu}(\mathrm{In}, \mathrm{Ga}) \mathrm{Se}_{2}$ solar cells

Bart Vermang, Jörn TimoWätjen, Viktor Fjällströma, Fredrik Rostvall, Marika Edoff, Rickard Gunnarsson, Iris Pilch Ulf Helmersson, Ratan Kotipalli, Frederic Henry, Denis Flandre

Thin Solid Films, 582, 300-303 (2015) 


\section{Acknowledgements}

I would like to first thank Iris Pilch for supervising me since I was a bachelor's student. You have taught me the importance of not jumping to conclusions too early and how to conduct research with good ethics. I would like to thank Daniel Söderström for supervising me in the beginning of my Phd studies. You sparked my interest for vacuum and plasma technology. A later addition to my supervisors was Nils Brenning, who deserves a big thanks. You have shown me the importance of not only pushing buttons in the lab, but to also try to understand the underlying physics behind what is going on. Lastly, my main supervisor Ulf Helmersson deserves a huge thanks. You gave me freedom to pursue what I found important to work on and without your enthusiasm to try new things, this nanoparticle synthesis process would have never been conceived.

There are also other people that have helped me that deserve to be mentioned. First a big thanks to Robert Boyd for his electron microscopy work which has helped my research. Thanks to Daniel Magnfält who always helps me when I do not understand something. Thanks to Petter Larsson for all the equipment you have created that has come to great use throughout my research.

Lastly a big thanks to all my colleagues and former teachers at IFM. 


\section{Table of contents}

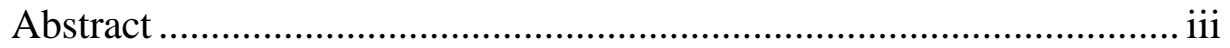

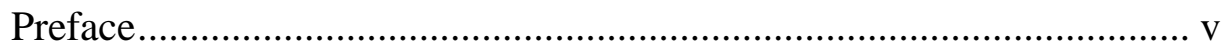

List of appended papers ................................................................ vii

Related Publications not included in this thesis ................................... ix

Acknowledgements .......................................................................... xi

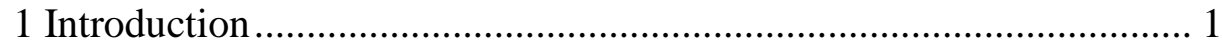

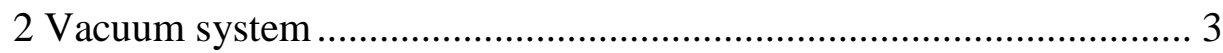

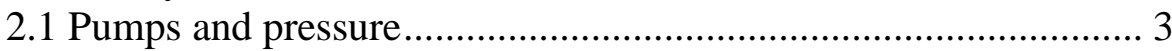

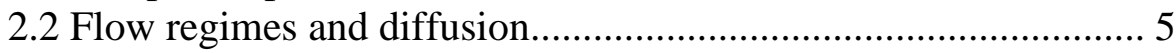

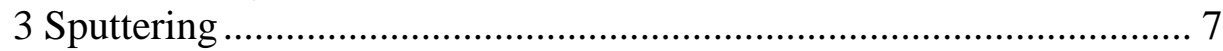

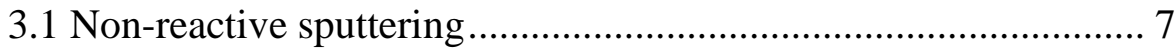

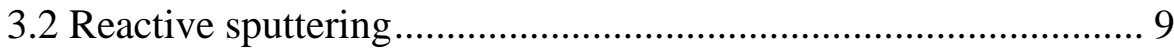

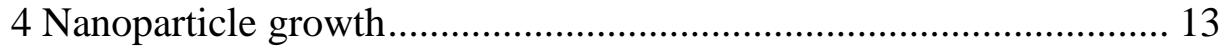

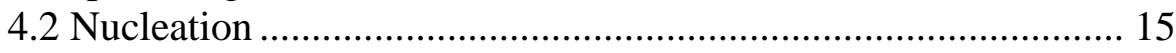

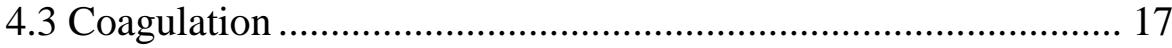

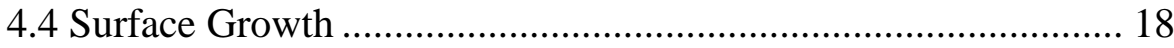

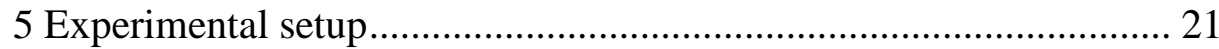

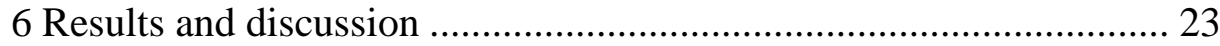

6.1 Influence of reactive gases on the cathode............................... 23

6.2 Influence of reactive gases on the nanoparticles.......................... 26

6.2.1 Influence on nucleation ..................................................26

6.2.2 Influence on oxygen content and crystal structure...........28

6.2.3 Oxygens influence on the particle size ..............................31

6.3 Size control .............................................................................. 31

6.3.1 Size control by changing the mesh bias ...........................32

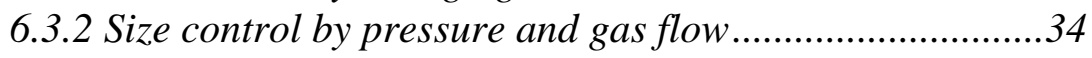

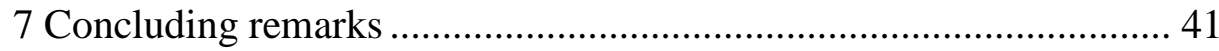

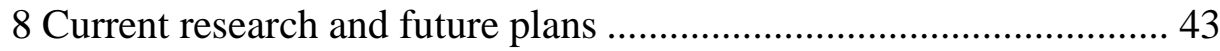

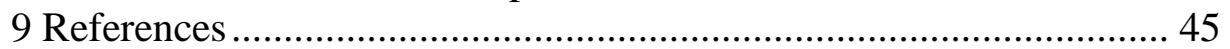




\section{Introduction}

Nanoparticles are one of the fundamental building blocks in nanotechnology which have the potential of greatly improving our life, health and environment. The reason nanoparticles are of such big interest compared to bulk materials is that their properties often depend on their size. For instance, a sunscreen that is based on titanium dioxide will have an increased protection against UV rays if the particle size is decreased [1]. One other size-dependent property is their surface area to volume ratio. Ten grams of $2 \mathrm{~mm}$ titanium dioxide particles will have an area of $71 \mathrm{~cm}^{2}$, while ten grams of $2 \mathrm{~nm}$ particles will have an area of $7100 \mathrm{~m}^{2}$. That is the size of a football field. A high surface area is important for gas sensing and catalytic reactions [2]. In addition to this, nanoparticles exhibit properties such as plasmonic resonances, which can enhance the efficiency of solar cells [3]. Nanoparticles are also dispersible in liquids and biological systems, which opens up doors for new diagnostics and treatments of cancer [4].

How well nanoparticles perform in these applications depends generally on their size, shape, composition and the amount of particles used. It is therefore crucial to have a nanoparticle synthesis process that allows for precise control over these properties. There are several ways of synthesizing nanoparticles. One easy way that anyone can do at home is to light a candle and hold a spoon close to the flame. The black soot that forms on the spoon will contain carbon nanoparticles [5]. One other method to obtain nanoparticle is to take a bulk object, and crush it into smaller pieces until it becomes nano-sized. These two methods are both simple and cheap, but they lack the required process control of the particle properties for them to be used in more sophisticated applications. On an industrial scale, wet chemistry is often used to produce nanoparticles. This is done by mixing a solution in a big batch where the nanoparticles nucleate and grow. Due to the complicated nature of mixing large batches, it is often challenging to control the size of the particles by this method [6]. In addition, if semiconductor grade particles are to be synthesized it is difficult to obtain a pure enough liquid media that do not contaminate the particles [7]. In this thesis a plasma-based synthesis method is used to grow nanoparticles. This process has previously shown promising results for nanoparticle size control [8] and fast nanoparticle 
growth [9]. However, it is still in its early stages of development and thus, fundamental understanding over the growth process and what parameters influences the nanoparticle properties is not yet fully explored. In addition to this, previous research on this process has only been published on copper nanoparticles. The use of a more reactive material such as titanium in an oxygen containing environment poses new challenges to the synthesis method. This thesis covers the influence of oxygen on nanoparticles synthesized from a titanium plasma. It also covers the influence of pressure, gas flow and electrical potentials on the resulting nanoparticle size.

In order to explain the results of this work, it is important to understand every parameter of the process that has been found to influence the particles. These topics has been divided up in to: vacuum system, sputtering and nanoparticle growth. Since it was found that residual gases present in the process had a profound influence on the nucleation of the particles and oxidation of the cathode which is the source of growth material, the topic of vacuum systems will be discussed first. Then the process of sputtering will be introduced and how this residual water and other oxygen containing gases influences the sputtering process. Lastly, this is tied together in a chapter about the nanoparticle growth which is dependent on the state of the cathode and the degree of vacuum achieved in the system. 


\section{Vacuum system}

The plasma based nanoparticle synthesis process is operated inside a vacuum system. This section covers the vacuum regimes used, different flow regimes, what residual gases are and how it is possible to reduce their partial pressure.

\subsection{Pumps and pressure}

Vacuum is a space where gas has been removed so that the pressure is reduced compared to the atmospheric pressure. It is however not possible to remove all gas molecules from an enclosed volume to create a perfect vacuum. Instead different degrees of vacuum is often used to define how low pressure that is achieved.

Table 1 Different vacuum regimes

\begin{tabular}{|l|l|}
\hline Degree of Vacuum & Pressure range $(\mathrm{Pa})$ \\
\hline Low & $10^{5}>\mathrm{P}>3.3 \cdot 10^{3}$ \\
\hline Medium & $3.3 \cdot 10^{3} \geq \mathrm{P}>10^{-1}$ \\
\hline High & $10^{-1} \geq \mathrm{P}>10^{-4}$ \\
\hline Very high & $10^{-4} \geq \mathrm{P}>10^{-7}$ \\
\hline Ultrahigh & $10^{-7} \geq \mathrm{P}>10^{-10}$ \\
\hline Extreme ultrahigh & $10^{-10}>\mathrm{P}$ \\
\hline
\end{tabular}

In Table 1, the different vacuum regimes that will be discussed in this thesis are presented. The lower the pressure is, the higher the vacuum is said to be. There are several factors that determine the lowest pressure that is possible to obtain. One factor is what pumps that are used. A positive displacement pump operate in the same way as a bicycle pump. As you pull the pump piston, the volume inside the pump increases which causes a pressure difference that makes air flow to fill this new volume. Then the gas is compressed and ejected to fill a bicycle tire and a new stroke of the piston repeats the cycle. In this analogy, the pressure in the room will decrease as the pressure in the tire increases. If this principle of pumping is used, no higher regimes than medium vacuum can be 
obtained. The reason for this is as the amount of gas atoms in the volume become less, they will collide with each other less frequently. Instead collisions with the wall of the vacuum system becomes more prominent. Since the atoms collide with the walls instead of with each other, a positive displacement volume will not be noticed, i.e the atoms will not push each other to fill this volume. In addition to this, there will be back streaming of gas from the pump that will contribute to the pressure inside the chamber. This low efficiency of positive displacement pumps at low pressure leads to that a second pump has to be added. Today, turbomolecular pumps are often put before the positive displacement pumps in order to compress the gas to a regime where the positive displacement pump can operate. With this setup it is possible to reach ultrahigh vacuum [10].

A turbomolecular pump backed with a positive displacement pump is used in this works experimental setup, but an ultrahigh vacuum is not reached. The main reason for this is that the vacuum system has to be opened on a regular basis to change the substrates. This does not give the vacuum system enough time to pump away the residual water adsorbed on the chamber wall. One other contributing factor is that the chamber is sealed with rubber gaskets which gases such as oxygen, nitrogen and water vapor have a relatively high permeation rate through. The contribution of these gases minus the gases removed by the pump, gives rise to the so called base pressure [10]. In the system used, a base pressure in the order of $10^{-4}-10^{-5} \mathrm{~Pa}$ is obtained. The dominating gas that gives rise to this pressure is water vapor.

Water vapor is a common problem in vacuum technology and there are several techniques that can be used to decrease its contribution to the base pressure. One method is to heat up the vacuum chamber, commonly referred to as baking. This increases the desorption rate of the water and initially increases the pressure of the system. When all water has desorbed, the pressure decreases to a lower level than before the baking procedure. Baking is however not as efficient on systems that have to be regularly opened and that are sealed with rubber gaskets. This is because the required baking time increases with decreasing temperature, and rubber gaskets are sensitive to high temperatures. One other technique to decrease the water vapor is to deposit a reactive coating inside the vacuum chamber. The desorbing water will land on this coating and bind 
to it which removes its contribution to the pressure [10]. This reactive coating is often titanium, which is also the material studied to make nanoparticles of in this thesis. Since titanium is highly reactive, the nanoparticle synthesis process will act as a pump that consumes water and binds it in to the particles.

\subsection{Flow regimes and diffusion}

The nanoparticle synthesis process cannot operate at the high vacuum regime because sputtering cannot be ignited [11] and particle nucleation does not occur at pressures of this low value. Because of this, argon gas is continuously flown through the system at a reduced pumping speed to increase the pressure. The total pressure of the system is given by the sum of the partial pressure of water, argon and other possible gases such as nitrogen, hydrogen and carbon dioxide. There are different flow regimes that behave differently depending on the pressure. The underlying reason for this change in behavior is due to that the mean free path of the molecules depends on the gas density. Equation (1) shows how the mean free path $\lambda$ is calculated.

$$
\lambda=\frac{1}{2^{1 / 2} \pi d_{0}^{2} n}
$$

Here $d_{0}$ is the molecular diameter and $n$ is the gas density in molecules per cubic meter. The flow regime is defined by the Knudsen number $K n$, which is the ratio between the mean free path and the diameter of the pipe $d$ that the gas flows through.

$$
K n=\frac{\lambda}{d}
$$

When $\mathrm{Kn}>1$ the flow is said to be molecular. This means that the pipe diameter is smaller than the mean free path and the gases collide with the pipe wall instead of with other gas molecules. This is the regime previously discussed, where positive displacements pumps do not work. In the region of $\mathrm{Kn}<0.01$ the flow is said to be viscous or continuous. This means that the collisions between gas molecules are more frequent 
than collisions with the wall and it is in this regime that the nanoparticle synthesis process operate in [10].

Even thou there are collisions between gas molecules, the gas density is significantly lower than at atmospheric pressures. This leads to that gas diffusion becomes a significant factor to consider when reactive gases is introduced to the process. The diffusion coefficient of two gases that interdiffuse is given by equation (3)

$$
D_{12}=\frac{0.019 T^{3 / 2}}{P \sigma_{12}^{2} \Omega}\left(\frac{1}{m_{1}}+\frac{1}{m_{2}}\right)^{1 / 2}
$$

Where $\mathrm{T}$ is temperature in Kelvin, $m_{1}$ and $m_{2}$ is the two different gas molecules mass, $\mathrm{P}$ is the pressure, $\sigma_{12}$ is the collision diameter and $\Omega$ is a dimensionless quantity [12]. For a pressure of $110 \mathrm{~Pa}$ and a temperature of $300 \mathrm{~K}$, the diffusion coefficient of oxygen in argon becomes 0.018 $\mathrm{m}^{2} / \mathrm{s}$, which is low enough that the inert gas flow of argon could be used to prevent oxidation of the cathode.

In summary, the process environment starts off in a high vacuum state where the pressure contribution is dominated by water vapor. Then argon is continuously flown at a decreased pumping speed to increase the pressure. At the higher pressure, the argon gas can be used as a shielding gas to reduce the flux of water vapor counter to the flow. The following section will cover how the growth material gets ejected from the cathode and how it interacts with the surrounding gas. 


\section{Sputtering}

\subsection{Non-reactive sputtering}

Sputtering is when a particle with mass such as an ion collides with a surface of a material and due to the collision, atoms from the material is ejected. The material can be either liquid or solid and is often referred to as a target [13]. The underlying mechanism behind the ejection of the sputtered material from a solid surface depend on the size of the ions used. Heavy ions colliding with the surface causes a cascade of collisions between target atoms. As the collision cascade develops, some of the collisions will be directed towards the cathode surface, which will knock out atoms. If a light ion is used instead of a heavy ion, sputtering will not occur from collisions with the surface. Instead the ion will first collide deeper inside the cathode material, and then strike out the surface material from the backside. For ions of intermediate size, such as argon, both mechanisms contribute to the amount of sputtered material [14]. The sputter yield is defined as the number of ejected target atoms per incoming ion [15]. This value depends on the angle, mass and kinetic energy of the incoming ion as well as what target material that is being used [14]. It is not only atoms that gets ejected from the surface during sputtering, secondary electrons are also released as the ions approaches the cathode surface. If these secondary electrons get accelerated by the negative potential of the cathode, they will obtain enough kinetic energy to further ionize the argon gas close to the cathode and this ionization process creates a plasma [16]. 


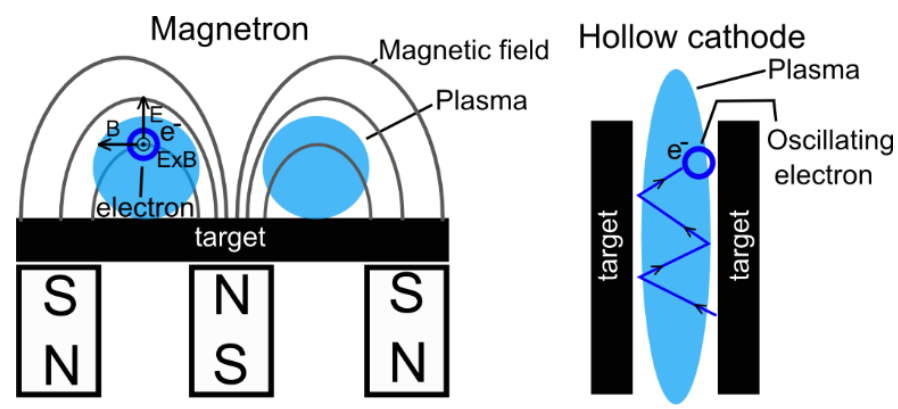

Figure 1: The different mechanisms for confining electrons in a magnetron and a hollow cathode

When using sputtering to synthesize nanoparticles, it is common to use a magnetron in a gas aggregation cluster source based on the Haberland concept [17]. A sputter magnetron is device where a magnetic field is present in the vicinity to the target surface. An illustration of a magnetron can be found in Figure 1. The purpose of the magnetic field is to confine the secondary electrons close to the target surface. The electrons will first be accelerated by the electric field. Then as the magnetic field becomes parallel with the cathode surface, their trajectory will bend towards the surface where they might be reflected again. This causes a hopping motion of the electrons where ionizing collisions with neutral atoms can occur [18]. This will locally increase the plasma density and since more ions are available, it helps to sustain the discharge at lower pressures [19]. However, a magnetron is not used in the present work, instead a hollow cathode is utilized. The hollow cathode is in this case a cylinder, where the discharge develops inside. The electron trapping inside a hollow cathode occurs by a different means than that of a magnetron. When high energy electrons gets released from the cathode surface it will first be accelerated by the cathode sheath and the repelled by the cathode sheath on the opposite side. This causes an oscillation of the electrons inside the hollow cathode. Collisions with these electrons will ionize the gas atoms, which results in a highly ionized plasma [20].

To further increase the ionization of the plasma, high powered electrical pulses has been used. This is done by applying square wave voltage pulses of a higher amplitude than would have been possible in direct current (DC) mode without melting the target [16]. 


\subsection{Reactive sputtering}

When a reactive gas that can chemically bind to the sputtered material is added, the process becomes a reactive sputtering process. A complex interplay between the inflow of reactive gas, oxidation of the cathode, reaction with the deposited material and the pumping speed of the vacuum chamber arises. The process can be characterized and understood by flowing oxygen to the process and monitoring its partial pressure. A so called hysteresis curve is then often found.

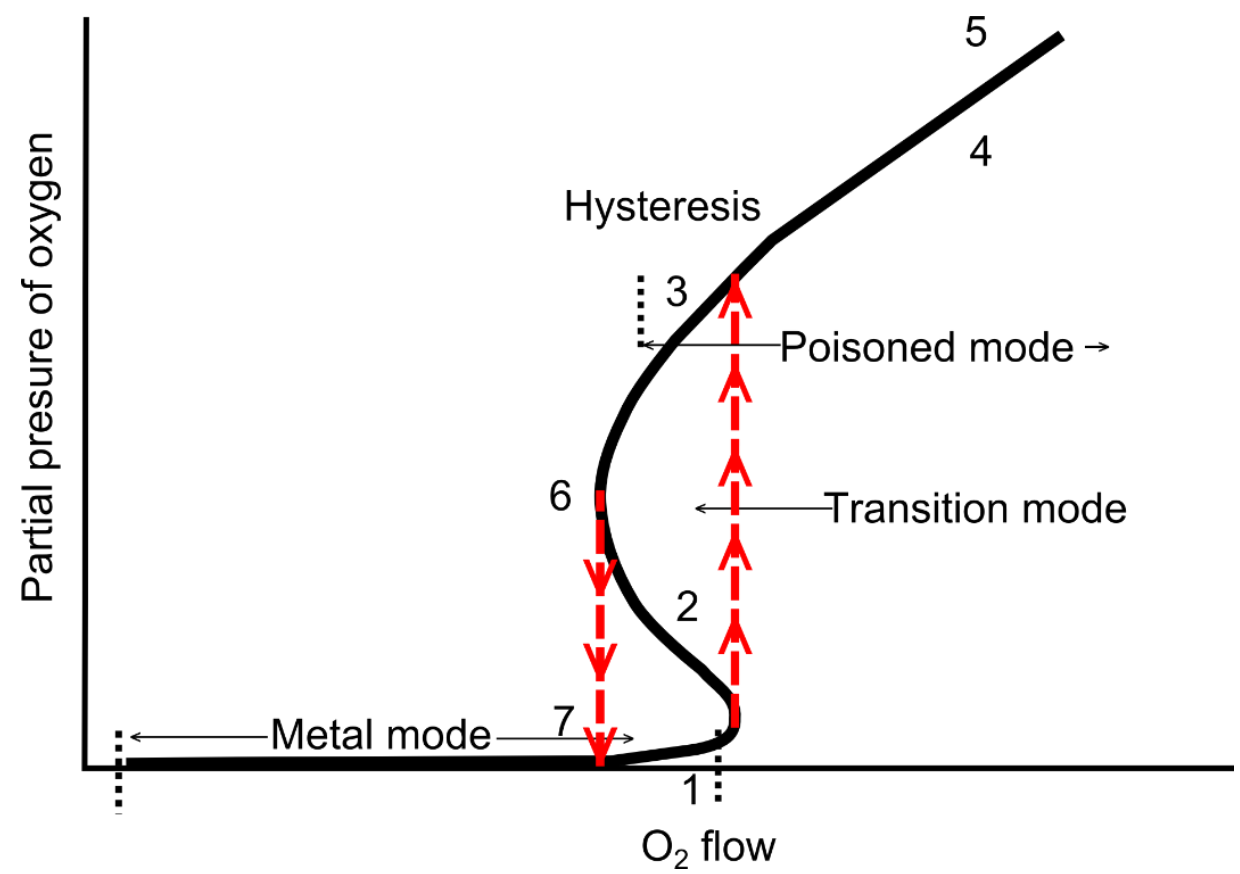

Figure 2: Sketch of a typical hysteresis in steady state (black line). If no fast feedback control is used, the partial pressure follows the red dashed lines from 1 to 3 when the oxygen flow is increased. Then it follows 6 to 7 when the flow is decreased. This exposes a hysteresis in the process

An illustration of a typical hysteresis behavior of a reactive sputtering process inside a vacuum system can be seen in Figure 2. The partial pressure of oxygen in the vacuum system is displayed on the y-axis, and the oxygen gas flow rate is displayed on the x-axis. At the area marked as metal mode, there is no significant increase in the partial pressure of 
oxygen, even though oxygen gas is continuously added to the process. The reason for this is that the oxygen gets consumed by the sputtering target, the sputtered material and the pump of the vacuum system. Since there is no or little oxygen bound to the target in this region, it is often referred to as the metal mode. As the oxygen is further increased to the point marked as 1 , there are three different points $(1,2,3)$ in the S-shaped curve that the partial pressure can have at steady state. It is however very difficult without an automatic fast feedback regulation loop to follow this S-shaped curve manually by tuning the flow rate and observing the partial pressure. This region is called the transition mode and a small perturbation such as an arc can make the cathode jump from position 1 to 2 or 3 . If the oxygen gas flow is increased to higher values than at position 1, the partial pressure follows the red dashed line and jumps directly to position 3. This region is called the poisoned mode, and the cathode is here fully oxidized. A further increase of the oxygen flow to the process linearly increases the partial pressure of the vacuum system. This is because no more oxygen can be consumed by the sputtering process. As the oxygen flow is decreased to 6 , the partial pressure follows the red dashed line to 7. A hysteresis between the two red dashed lines is then observed [19]. The reasons for this hysteresis is that the cathode is already oxidized at position 6 and the deposition of titanium is less than at position 1 . When the cathode clean sputters itself the partial pressure of oxygen decreases as the cathode becomes more efficient at sputtering out titanium which consumes oxygen.

It is often desired to operate the cathode inside the transition regime, since the deposition rate is much higher compared to the poisoned regime. One other reason is that in the poisoned mode, the grown film can only be fully stoichiometric, but it is possible to deposit sub stoichiometric films in the transition and metal mode [21]. Since it is difficult to have a stable transition mode, it is difficult to control the film stoichiometry. Several techniques have previously been used to try to stabilize the process in the transition mode when depositing thin films with a magnetron. Smaller cathode surface areas has been shown to be more stable [22]. Using high powered pulsed sputtering has also been shown to stabilize this zone in certain parameter ranges [23] [24]. The utilization of a hollow cathode where inert gas flows through it in combination with separate injection of reactive gas from the cathode surface has given more stability of the 
reactive sputter process [25]. In paper $\mathbf{1}$, all of these beneficial effects are combined and a stable process in the transition regime was possible.

In the work in this thesis, the power supply that feeds DC voltage to the pulsing unit is operated in a constant average current mode. This leads to that the cathode discharge voltage changes when oxygen is introduced to the sputtering process. The main reason for this has been found to be due to a change in the secondary electron emission yield as the target gets oxidized [19]. The discharge voltage can be used to indicate the oxidation state of the cathode, but careful considerations of other parameters that influence the voltage has to be made. An increased voltage for a constant current leads to a higher average powers. Since a pulsed voltage is used another phenomenon also arises when oxygen is added to the sputtering process of titanium, and that is that the peak current value increases for a constant average current [26]. 



\section{Nanoparticle growth}

It has now been explained how the growth material gets ejected, and how the ejection of the growth material depends on the oxidation state of the cathode. In this section we will look closer on how this growth material is transformed into nanoparticles. Since the growth stages depend on the charge of the particles, a short introduction to how particles charge up in a plasma will first be given. This is followed by a discussion about how the particles nucleate in the plasma. Lastly, the growth by coagulation and through surface growth will be explained.

\subsection{Particle charging}

If a particle is put inside a low temperature laboratory plasma it will typically acquire a negative charge. This is because the electrons in the plasma have a higher velocity than the ions which gives them a higher probability of colliding with a particle. As the particle has acquired a negative charge, it will start to repel electrons and attract ions. A positive ion sheath forms around the negatively charged particle. This sheath length is related to the Debye length, $\lambda_{D}$ which is the distance where the negative charge of the particle is fully shielded by the positive ions [27].

$$
\frac{1}{\lambda_{D}^{2}}=\frac{n_{e 0} e^{2}}{\varepsilon_{0} k_{B} T_{e}}+\frac{n_{i 0} e^{2}}{\varepsilon_{0} k_{B} T_{i}}
$$

Where $n_{e}$ is the electron density $n_{i}$ is the ion density, $\mathrm{T}_{\mathrm{e}}$ is the electron temperature and $\mathrm{T}_{\mathrm{i}}$ is the ion temperature, e is the elementary charge, $\varepsilon_{0}$ is the dielectric constant of the vacuum and $k_{B}$ is the Boltzmann constant [28]. The charge of a particle is determined by the balance of the electron and ion currents. The dust particles negative charge reduces the electron current, so that the particle obtains no net currents at steady state. The correct equations to describe the ion and electron current to the particle depend on the size of the particle relative to the Debye length. If the particle is small (or the Debye length is long) $\left(\lambda_{D}>>\right.$ a), the ions will start to orbit around the negatively charged particle and hit it with an incidence angle. The theory used to describe this is called Orbital Motion Limited (OML) [27]. To get the OML current in the case for a Maxwellian 
velocity distribution it is necessary to integrate for all velocities from every charge contribution for the full solid angle $4 \pi$ [28].

If the particle is large enough to obtain the floating potential of the plasma, the the electron (5) and ion (6) flux to the particle is given by:

$$
\begin{gathered}
I_{e}=-\pi r^{2} n_{\mathrm{e}} e \sqrt{\frac{8 k_{B} T_{e}}{\pi m_{e}}} \exp \left(\frac{e V}{k_{B} T_{e}}\right) \\
I_{i}=\pi r^{2} n_{i} e \sqrt{\frac{8 k_{B} T_{i}}{\pi m_{i}}}\left(1-\frac{e V}{k_{B} T_{i}}\right)
\end{gathered}
$$

Where $r$ is the particle radius $V$ is the floating potential, $m_{i}$ is the ion mass and $m_{e}$ is the electron mass [27]. If the particle radius is much shorter than the Debye length $\left(\lambda_{D}>>r\right)$, a different equation has to be used which is covered in the surface growth section of this chapter.

Due to the electron and ion currents, the particles in a low temperature laboratory plasma will on average obtain a negative charge. However, if one look at a single particle, the charging is a discrete process [27]. This becomes significant for particles in the size range of 1-2 $\mathrm{nm}$ in diameter, since such small particles can only hold up to one or two charges [29] [30]. This means that particles can obtain neutral, or even in some special cases positive charge if they statistically just happen to collide with more ions [27].

Lastly it shall also be noted that the particle charge depends on the number density of particles in the plasma. By looking at the condition for quasi neutrality:

$$
n_{e}=n_{i}-Z_{p} n_{p}
$$

Where $Z_{p}$ is the particle charge and $n_{p}$ is the number density of particles in the plasma. For this quasi neutrality to hold when the total particle charge density $Z_{p} n_{p}$ approaches the same order of magnitude as the charge density of ions $n_{i}$, the plasma will get depleted of electrons, reducing $n_{e}$. Since the particles obtain their charge from the current from 
this depleted regime, the absolute value of the particles floating potential will decrease [31].

\subsection{Nucleation}

In this section, the physical mechanisms behind the first stages of the nanoparticle growth called nucleation is covered. Nucleation has been defined as when a sub critical cluster has become large enough to continue to grow [27]. There are also different types of nucleation mechanisms. The same definitions as used by Bouchoule in ref [27] will from here on be used. Homogenous nucleation is when only neutral monomers or clusters of atoms are involved. Ion induced nucleation is when ions and all the consequences of charges are involved. When sub critical clusters are created by chemical reactions, it is referred to as chemical nucleation [27]. The Gibbs-Kelvin equation (8) can be used to thermodynamically determine the critical diameter for which a small particle is stable in a super saturated vapor. Particles of sizes less than $d_{c r i t}$ will evaporate and disappear. This equation is only valid for macroscopic particles that exhibit properties such as surface tension. However it should be noted that even though this equation cannot be used to describe it, under certain circumstances the critical diameter can be less than the size of two atoms [32].

$$
d_{c r i t}=\frac{\gamma V_{m o l}}{k_{B} T \ln (S)}
$$

The critical diameter depends on the surface tension $\gamma$, the molecular volume $V_{\text {mol }}$, the temperature $\mathrm{T}$, and the super saturation ratio $\mathrm{S}$ [32].

The nucleation of nanoparticles from a sputter source is still a phenomenon that is being studied [33] [34] [35] [36]. For the synthesis of titanium, cobalt [33] and tungsten [37] nanoparticles it has been found to be necessary to have a partial pressure of reactive gas such as oxygen or water vapor in order for particles to nucleate. For the synthesis of copper nanoparticles, oxygen was not found to be necessary, but at certain partial pressures it increased the nanoparticle mass deposition rate [38]. The explanation for this behavior was attributed to the different binding energies between metal-metal dimers and metal-oxygen dimers. A titanium-titanium dimer has a low binding energy of $1.219 \mathrm{eV}$, while a 
titanium-oxygen dimer has a high binding energy of $6.908 \mathrm{eV}$. For the case of copper, the copper-copper dimer binding energy is $2.0834 \mathrm{eV}$ while the copper-oxygen dimer is not much higher with a binding energy of $2.974 \mathrm{eV}$. In addition to this, the sputter yield of copper is much higher than that of titanium, which gives a higher density of copper atoms that leads to a higher probability of two copper atoms colliding with each other [33]. Even though the density of the sputtered material in a pulsed hollow cathode can reach orders of $10^{19}-10^{20}$ ions $/ \mathrm{m}^{3}$ [16] no particles was found in paper 2 at standard parameters when the partial pressure of contaminants was too low. It is thus obvious that the same reaction mechanisms described for the nucleation of copper particles cannot be used to describe how the majority of titanium particles nucleate. Copper can nucleate through homogenous nucleation. For this to happen, the inert process gas has an important role in the nucleation process. The gas works as a cooling media that dissipates heat when atoms bind together. By looking kinetically on the nucleation mechanism, with only monoatomic species involved, two metal atoms and at least one argon atom has to collide at the same time in order for a stable dimer to form. The reason for this is that the binding energy needs to be dissipated by a collision with an argon atom that carries away this excess energy. Without the argon atom, the molecular bonds in the dimer would split apart from its obtained thermal energy when binding together. These types of collisions are called three-body collisions, and is often used to describe homogenous nucleation [35]. The exact reaction mechanism for the chemical nucleation of the titanium nanoparticles synthesized in this thesis is today unknown. The explanation becomes further complicated, since most of the growth material is ionized, and oxygen can form negative ions that might play an important role. It has also been suggested that oxide formation on the cathode surface could cause small arcs that eject stable clusters of atoms which can grow to larger particles [38].

From equation (8) it can be seen that the amount of sputtered material is an important factor that contributes to the super saturation ratio and thus the critical size. Since the sputter yield of metal from the cathode decreases when oxygen reacts to it [21], oxygen can thus decrease this super saturation ratio and reduce the particle nucleation rate. It has been observed that there is an optimum value of the particle mass deposition rate that depends on the cathode discharge power and the oxygen gas flow. However this high deposition rate was only stable in a narrow 
process window [34]. This concludes that in order to get a good and reliable process both the oxidation state of the cathode and the oxygen content of the vacuum system have to be precisely controlled.

The inert gas pressure is also an important parameter that has to be considered for the particle nucleation. It has been reported that homogenous nucleation of vanadium nanoparticles was made possible by increasing the argon gas pressure. The higher inert gas pressure allowed the metal vapours to cool down more efficiently. However, the base pressure during these experiments were in the $10^{-5} \mathrm{~Pa}$ range. This means that there are still a significant amount of contaminants that could have contributed to chemical nucleation of the particles [39]. The same phenomenon was observed in paper 2 but also an increase in the particle oxygen content and cathode voltage was observed with increasing inert gas pressures, which suggests that oxygen was still contributing to the nucleation.

\subsection{Coagulation}

In this section, the growth of nanoparticles through inelastic collisions will be covered. This process is called coagulation, and it is when species larger than a monomer collide with each other and bind together [32]. This coagulation theory was made for capacitively coupled argon-silane discharges, and the sizes of particles obtaining the floating potential of the plasma might vary in the hollow cathode plasma discharge [27]. Theoretical work has also been made on the coagulation in expanding copper plasmas produced by lasers evaporation where it was found to have a small influence on the resulting particle size [40].

When the number density of particles is high, growth through coagulation can occur [40]. Since the particles are inside a plasma, they will be charged up by collisions with ions and electrons. A small particle with a size of 1-2 nm does not hold more than one or two charges [29] and thus if a negatively charged particle collides with two ions its charge can change sign to positive. This leads to that small particles in the plasma can have negative, neutral and positive charges. Negative and positive charged particles will then be attracted to each other and bind together. There will also be an attractive force between charged and neutral particles due to an image potential induced by the charged particle. As 
the particles grow larger, they will obtain an increasingly negative charge, and coagulation will eventually be suppressed due to coulomb repulsion [30]. It is still however possible that this coulomb repulsion could be surpassed if the particles have a high kinetic energy. And the Coulomb repulsion could be reduced if the density of nanoparticles are high enough to cause electron depletion in the plasma that decreases the particle charge.

\subsection{Surface Growth}

When the coagulation stops and the particles obtain a negative charge, the flux of ions from the OML current will be the main contribution of growth material to the particle. Why this is the case can be understood by looking at the collision cross section for ions and neutrals on a charge particle. The collision cross section of a neutral is given by

$$
\sigma_{\text {neutral }}=\pi r^{2}
$$

Which is the same as the geometrical cross section. By using a simplified equation for the floating potential

$$
V=-\frac{K_{1} k_{B} T_{e}}{e}
$$

Where $K_{l}$ is a function of the ion mass and the ratio between the electron and ion temperature. For titanium and in the parameter regime worked in, a value of $K_{l}=2.48$ can be used. The collision cross section for a positive ion on a negatively charged is then given by

$$
\sigma_{\text {ion }}=\pi r^{2}\left(1+K_{1} \frac{T_{e}}{T_{i}}\right)
$$

The collision cross section will thus be much larger, in the order of two magnitudes for ions compared to neutrals [8]. This has shown to give growth rates in the order of $470 \mathrm{~nm} / \mathrm{s}$ when a copper hollow cathode was used [9].

The electron and ion currents will cause heating of the particle. And the mechanism through which the particle cools down is by radiating heat or by collisions with colder gas atoms. This is significant in high density 
plasmas, since there are less gas atoms that can cool the particle and a higher ion current that heats the particles which could lead to evaporation of particles [27]. 



\section{Experimental setup}

Two slightly different experimental setups were used in the appended papers. In paper 1, a stainless steel mesh (Figure. 3 a) was used around the growth zone of the particles. The exact function of this mesh is still unknown, however it has been found to increase the reproducibility of getting particles down on the substrate, compared to not using anything at all.

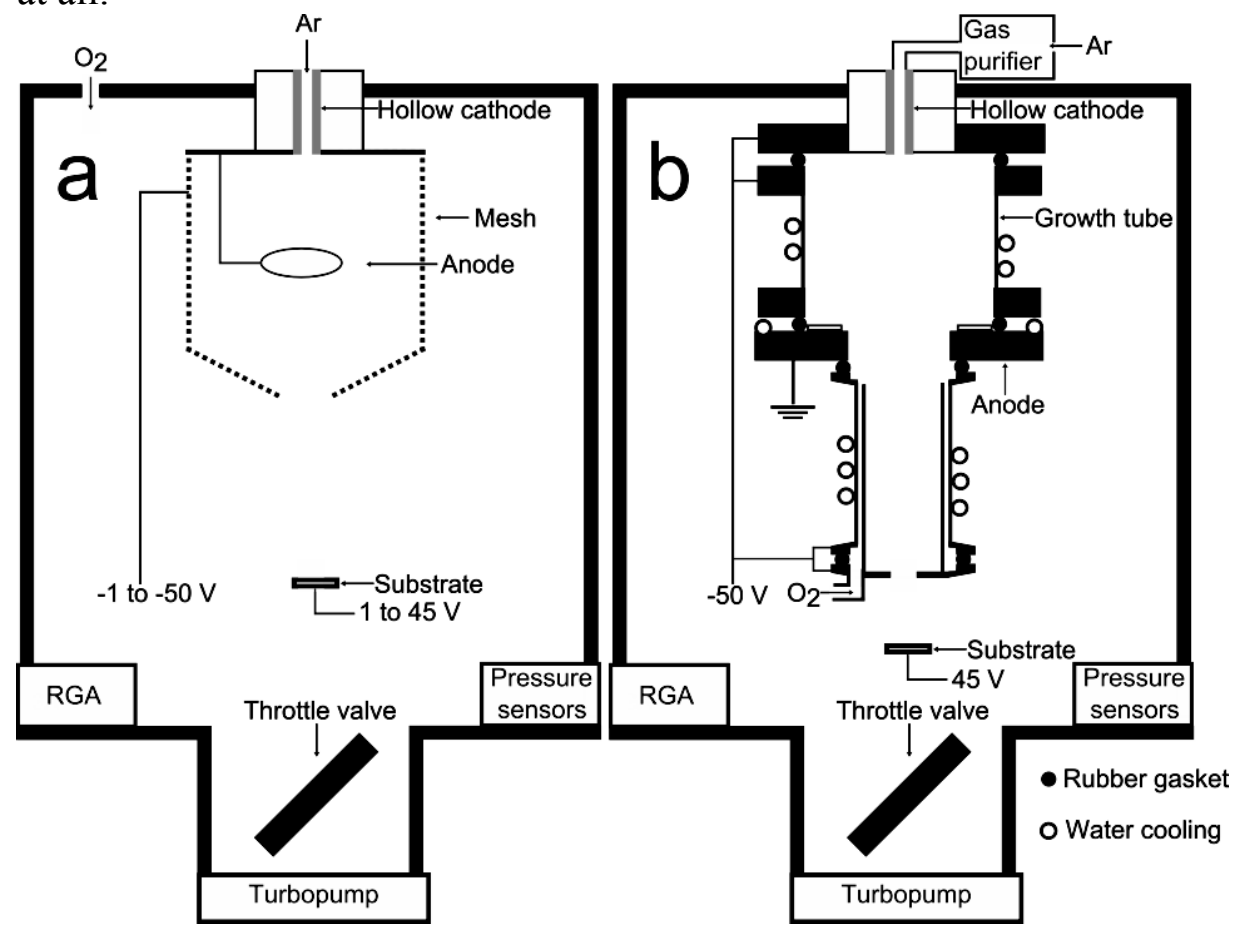

Figure 3: The experimental setups used. With the mesh and anode ring (a) used in paper 1 . The experimental setup with the growth tube (b) used in paper 2.

A negative bias voltage can be put on the mesh, and if the potential between the mesh and the cathode becomes higher than the mesh and anode, a portion of the discharge current can flow through it. Another difference in (a) is the positioning and shape of the anode. Here it is made out of a thin ring suspended on a wire. There were several problems with the experimental setup that needed to be addressed for the experiments made for paper 2 . The main problem was low reproducibility between 
experiments which manifested itself as drifts in the discharge voltage and the particle size distributions as well as uninterpretable x-ray diffraction patterns for particles produced without the addition of oxygen. This was believed to be mainly associated with fluctuations of the residual water in the vacuum system. To minimize the effects of the base pressure, a tube was put around the particle growth zone to prevent water vapour from diffusing in to the plasma and react with the particles and cathode. Several prototypes of this growth tube were tested and it was found that a tube with diameter of $40 \mathrm{~mm}$ was too narrow to use up in the expansion zone of the plasma. A tube of diameter $73 \mathrm{~mm}$ and length of $68 \mathrm{~mm}$ gave a sufficient expansion zone, that could be later narrowed down to $40 \mathrm{~mm}$ in the bottom part of the tube (Figure $3 \mathrm{~b}$ ). To focus the particles with the gas flow and to further suppress inflow of water the exit hole of the growth tube was narrowed to a diameter of $10 \mathrm{~mm}$. To make sure that the argon gas that was fed to the process was clean, a gas purifier was put on the gas line. Water cooling of the growth tube was necessary, since the discharge voltage drifted with temperature. With this setup, it was possible to reach a clean state where particles did not nucleate without the addition of oxygen. This is why there is an oxygen inlet in the lower part of the growth tube. Injecting oxygen outside of the system as in (a) did not influence the discharge inside the growth tube.

Other than these differences, the experimental setups are similar. It is possible to change the negative potential on both the growth tube and mesh to allow for size control. The chamber pressure at constant gas flow is set by restricting the pumping speed with the throttle valve. A differentially pumped residual gas analyser is used to monitor the partial pressure of the gases in the chamber. The substrates have a positive potential to attract the particles which have a negative charge

The discharge parameters used in both papers were similar and is here by referred to as the standard parameters. A frequency of $1500 \mathrm{~Hz}$ was used with a pulse width of $80 \mu \mathrm{s}$. The power supply was operated in current regulation mode, and a constant current was set so that the average power to the cathode did not exceed $100 \mathrm{~W}$ when oxygen was added to the process. The average power to the cathode was around $93 \mathrm{~W}$ in the metal mode. This resulted in peak current values of around $10 \mathrm{~A}$ and discharge voltages in the order of $280 \mathrm{~V}$. 


\section{Results and discussion}

In this chapter, the results from the two papers are tied together and discussed combined with un-published results obtained from sputtering a molybdenum cathode. First, the influence of the reactive gas on the process and the particles will be presented. Then different means of controlling the particle size will be discussed.

\subsection{Influence of reactive gases on the cathode}

The influence of reactive gases on the nanoparticles and the synthesis process was prior to this work unknown for this pulsed hollow cathode synthesis process. However, for the synthesis of titanium, it was found to be one of the most important process parameter. Here, all the important findings are presented with some additional un-published data.

First we will look at the influence of oxygen flow to the chamber on the discharge voltage and the oxygen partial pressure. This experiment was done with the mesh (Figure. 3 a), so the oxygen could react with the plasma. 


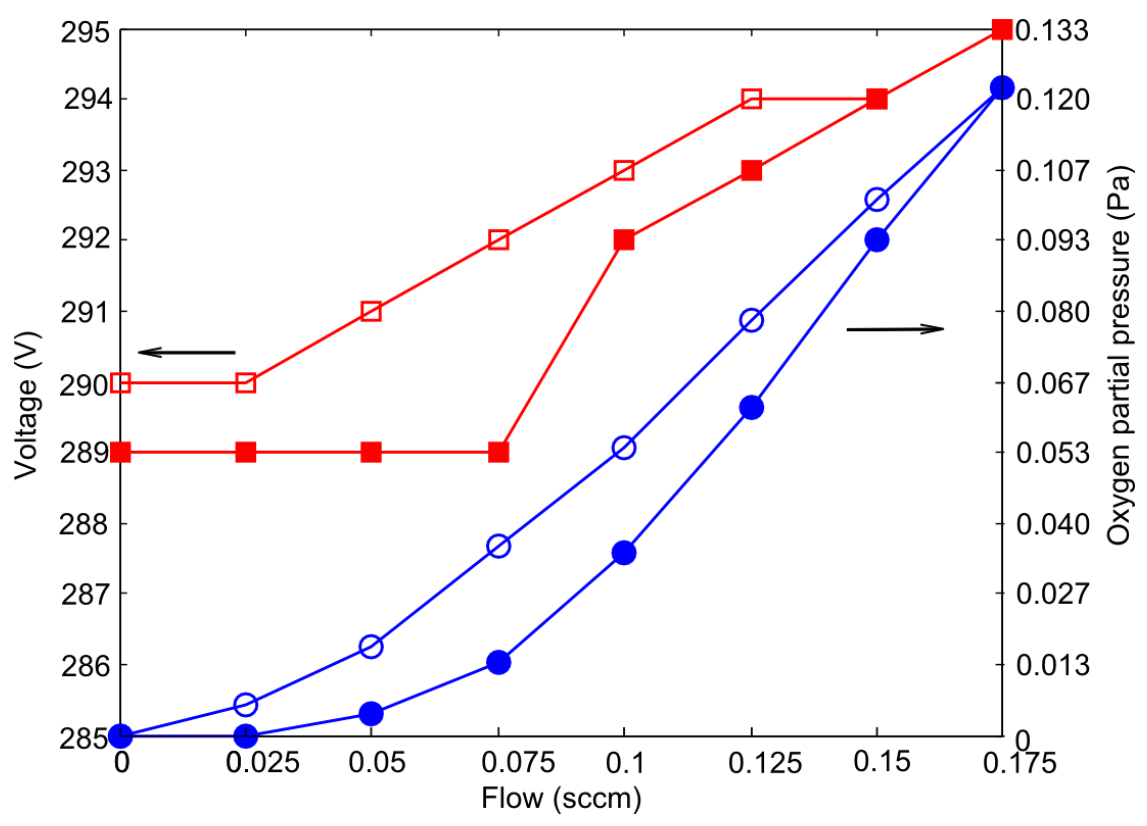

Figure 4: Oxygen partial pressure (blue circle) and cathode discharge voltage (red square) as a function of oxygen gas flow. The cathode voltage and the partial pressure increases with increasing oxygen gas flow (filled markers). When the oxygen gas flow decreases (open markers), a small hysteresis is found.

In Figure. 4 it can be seen from the red curve that the cathode indeed reacts with the oxygen which changes its discharge voltage. It should be noted that the plateau at $289 \mathrm{~V}$ was not always there and sometimes the voltage change was observed at lower gas flows than $0.075 \mathrm{sccm}$. This implies that to only have an inert gas flow through the hollow cathode does not fully suppress oxygen from reacting with it. At the highest reactive gas flow of $0.175 \mathrm{sccm}$, the cathode is still in the transition mode and a further increase would increase the discharge voltage. When the gas flow is decreased (open markers) a hysteresis in the discharge voltage can be seen. By looking at the blue curve which shows the oxygen partial pressure of the chamber, a small hysteresis is also observed. However, this hysteresis is nothing like the ones typically seen in magnetron discharges, and if a longer waiting time between the measurement points were made, the hysteresis vanished. To grow fully stoichiometric titanium dioxide nanoparticles, it was not necessary to use higher gas flows than $0.125 \mathrm{sccm}$, at an argon gas flow of $90 \mathrm{sccm}$. This means that the cathode can be operated in the transition mode while still producing 
fully stoichiometric particles which allows for a higher deposition rate. It should however be noted that the deposition rate decreased when fully stoichiometric titanium dioxide nanoparticles were synthesized, but no quantification of the magnitude has been made. The deposition rate further decreased for even higher oxygen flows, which is also evidence for that the cathode had not yet reached the poisoned mode when fully stoichiometric titanium dioxide particles where produced.

If the same experiment was run with the growth tube attached, the discharge voltage (red square) and partial pressure (blue circle) followed the curves in Figure. 5. It is clear that the discharge voltage does not get affected by the partial pressure of oxygen outside of the growth tube.

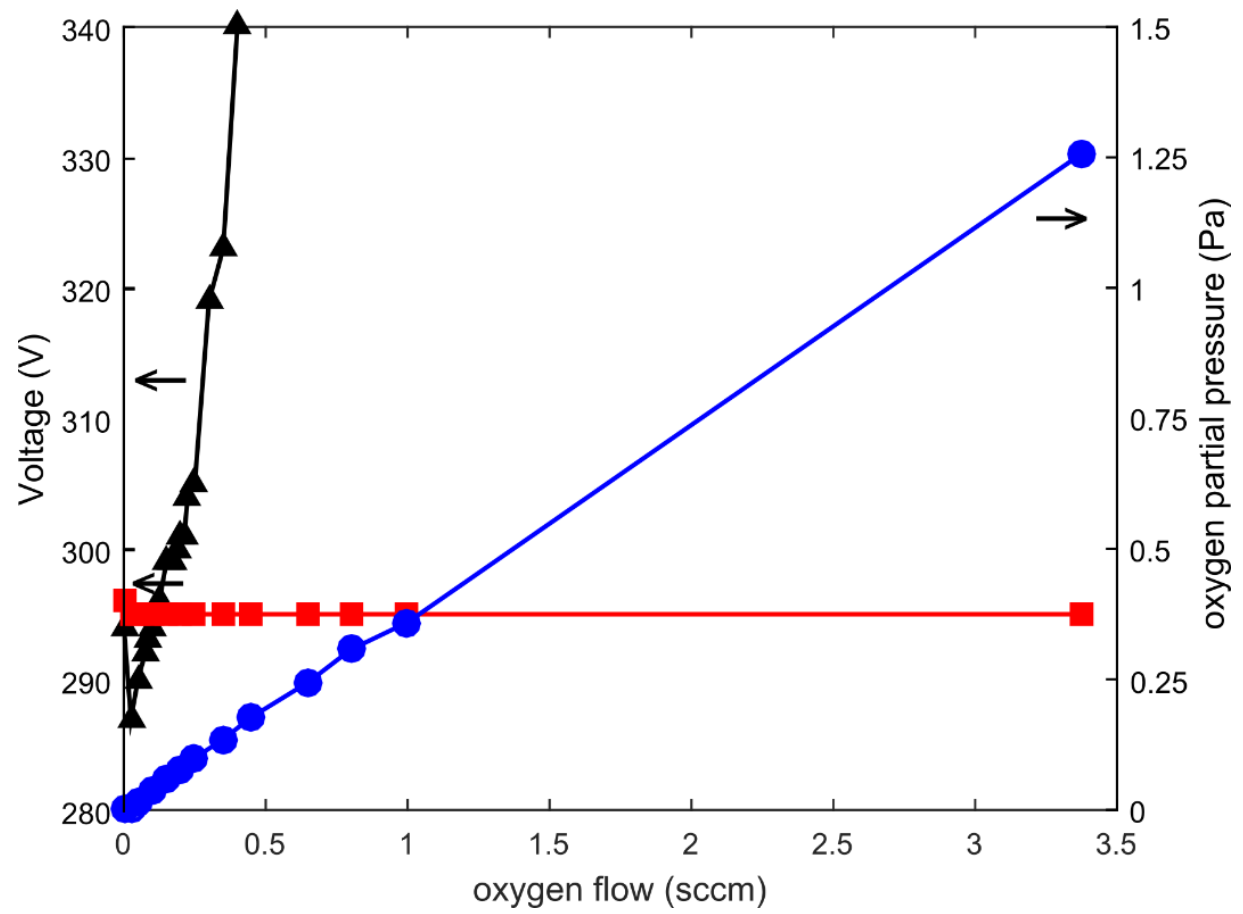

Figure 5: Oxygen partial pressure (blue circle) vs cathode discharge voltage (red square) as function of oxygen gas flow with the growth tube. The cathode discharge voltage without the growth tube is marked as black triangles.

It is thus safe to assume that the growth tube protects the cathode for oxygen partial pressures of at least $1.25 \mathrm{~Pa}$ at an argon gas flow of 90 
sccm. For comparison, the discharge voltage without the growth tube is plotted (black triangle) in the same graph. Already at $0.4 \mathrm{sccm}$, the cathode started to arc and the experiment had to be stopped. This result show that any fluctuation of the base pressure outside of the growth tube can be neglected. The only contaminants that could react with the particles is thus the ones desorbed from the inside of the growth tube. This led to a higher reproducibility and also the possibility to operate the discharge at clean conditions where the oxygen content was too low for nucleation to occur.

\subsection{Influence of reactive gases on the nanoparticles}

In this section, a closer look is taken on the influence of the reactive gas on the nanoparticles that are being produced. First the regimes where nucleation was possible is presented. Then the different crystal structures and the oxygen stoichiometry obtained are looked at. Lastly a discussion is made regarding whether the oxygen influences the particle size.

\subsubsection{Influence on nucleation}

With the growth tube present, it was possible to operate the process in regimes where no nucleation occurred unless oxygen was intentionally introduced in to the growth tube. This regime was present at high gas flows and low inert gas pressures. A plot of the regime which was experimentally found can be seen in Figure. 6, where the pressure is plotted on the $\mathrm{x}$-axis and the argon gas flow is plotted on the y-axis. At the parameters marked as circles, particles were found. At the parameters marked with crosses, no particles were found unless oxygen was added. This is interpreted as that chemical nucleation occurs below the pink dashed line, and above it, the partial pressure of residual gas $p_{o x}$ is too low. In this reasoning we assume a constant supply of reactive gas from the growth tube walls $Q_{o x}$. When the gas flow $Q_{A r}$ is increased, the reactive gases gets more diluted. When the pressure $p_{A r}$ is increased, the pumping speed that removes reactive gases from the growth tube becomes less. This gives us the relationship:

$$
p_{o x} \propto Q_{o x} \frac{p_{A r}}{Q_{A r}}
$$




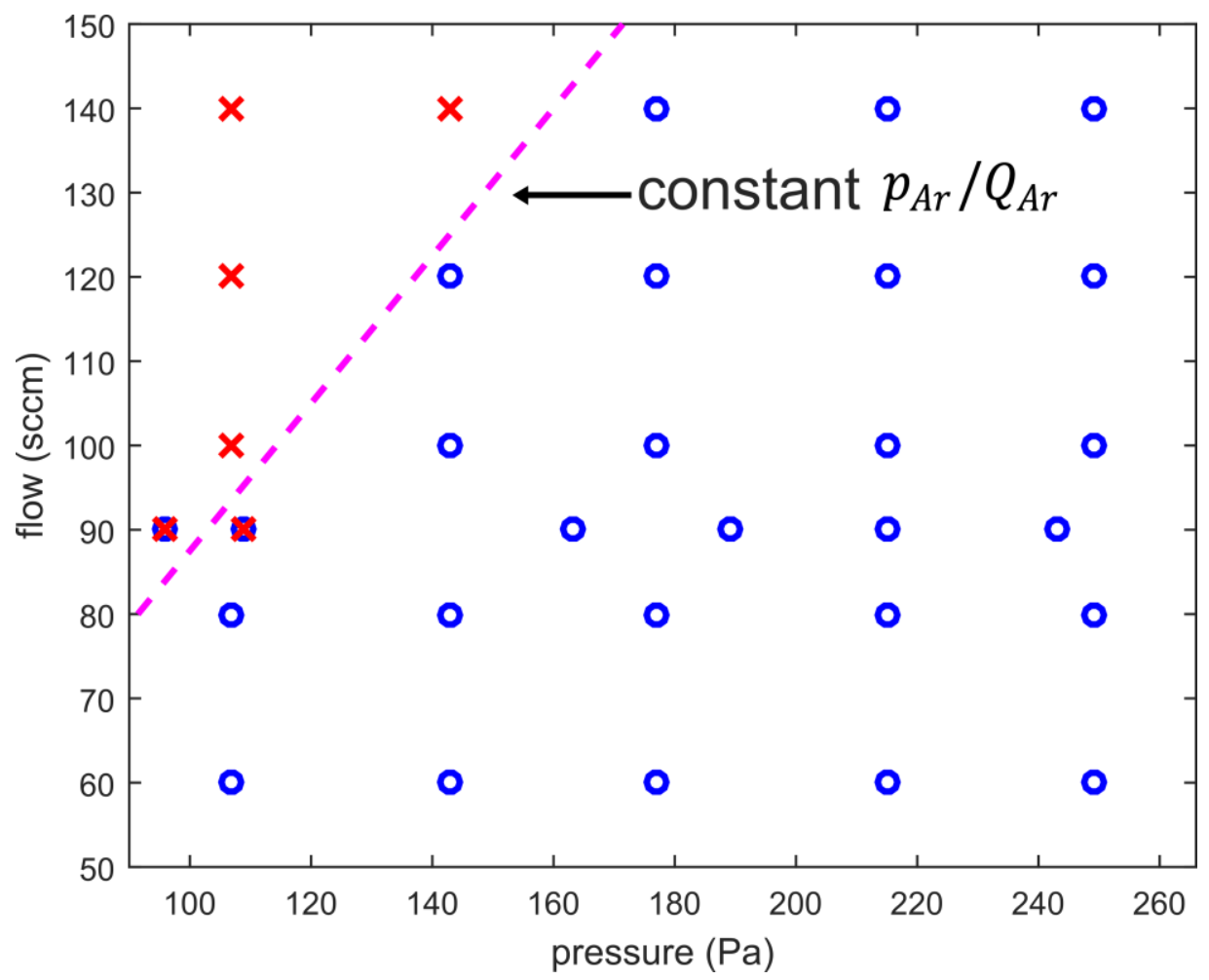

Figure 6: Nucleation of particles as a function of pressure and gas flow. Nucleation was possible without addition of oxygen (circles). Nucleation was not possible without the addition of oxygen (crosses). The dotted pink line shows where it is where it is a constant supply of contaminants for a constant base pressure.

Thus a line can be plotted in Figure. 6 (pink dashed) which was experimentally found to be valid for a base pressures between $4.5 \cdot 10^{-5}$ and $6.7 \cdot 10^{-5} \mathrm{~Pa}$. The line will also be shifted between experiments if the base pressure changes or during experiments if the particle synthesis process consumes the residual gas in the growth tube. This can be seen in the figure as positions marked with both a circle and a cross. Temperature fluctuations should also shift this line since the desorption rate $Q_{o x}$ depends on the temperature [10].

This interpretation assumes that homogenous nucleation is not possible in the regime studied, and the pink dashed line represents the limit due to the lack of chemical nucleation sites. However, it has been argued that an 
increased inert gas pressure could change the nucleation regime of vanadium nanoparticles from a chemical to a homogenous [39]. To answer the question if this is the case for titanium nanoparticles, more experiments in a much cleaner environment are necessary.

Nitrogen was also tested as a reactive gas for titanium to nucleate on. The cathode discharge voltage changed, but no particles were found on the substrate.

\subsubsection{Influence on oxygen content and crystal structure}

First we will look at the low reactive gas partial pressure regime, where the nanoparticles oxygen content most likely came from the residual water in the vacuum system. Repeated X-ray diffraction measurements on samples synthesized in this regime gave inconclusive results. Only once a peak of titanium together with peaks of titanium oxide could be identified. In addition to this, no pure titanium core and oxide shell on the titanium nanoparticles were ever found. This is different from the nanoparticles synthesized that can be found in the literature. A metal core and an oxide shell is found when synthesizing copper [41] and iron with the pulsed hollow cathode sputtering process. In a cluster source a titanium core and an oxide shell has been observed [42]. However, in a plasma gas condensation cluster deposition system, nanoparticles without any core shell structure were synthesized. It was argued that the lattice miss match between $\mathrm{NaCl}$-type titanium oxides and fcc titanium is less for nanoparticles compared to bulk. This led to that oxygen atoms could subsidize titanium atoms in the fcc lattice. It was also found that for particle sizes larger than $15 \mathrm{~nm}$ that were made out of $\mathrm{TiO}_{x}$, with a hcp phase, no oxide shell was formed. The explanation for this was that it was due to a high solubility of oxygen in the lattice [43].

The lack of a shell in this work could be due to that the oxygen content of the nanoparticles were too high so that a NaCl-type titanium oxide was always formed. When the oxygen content of the process was reduced, the particles stopped nucleate and thus lower oxygen containing nanoparticles could not be studied. This highlights one of the issues when synthesizing nanoparticles with this process. The issue is that clean 
particles cannot be made without stopping or reducing the nucleation. This was realized when trying to synthesize InAlN particles for a separate project, but only InAlON particles could be produced.

Moving on to higher oxygen contents, where oxygen was intentionally introduced to the process. X-ray diffraction of the nanoparticles synthesized is presented in Figure. 7.

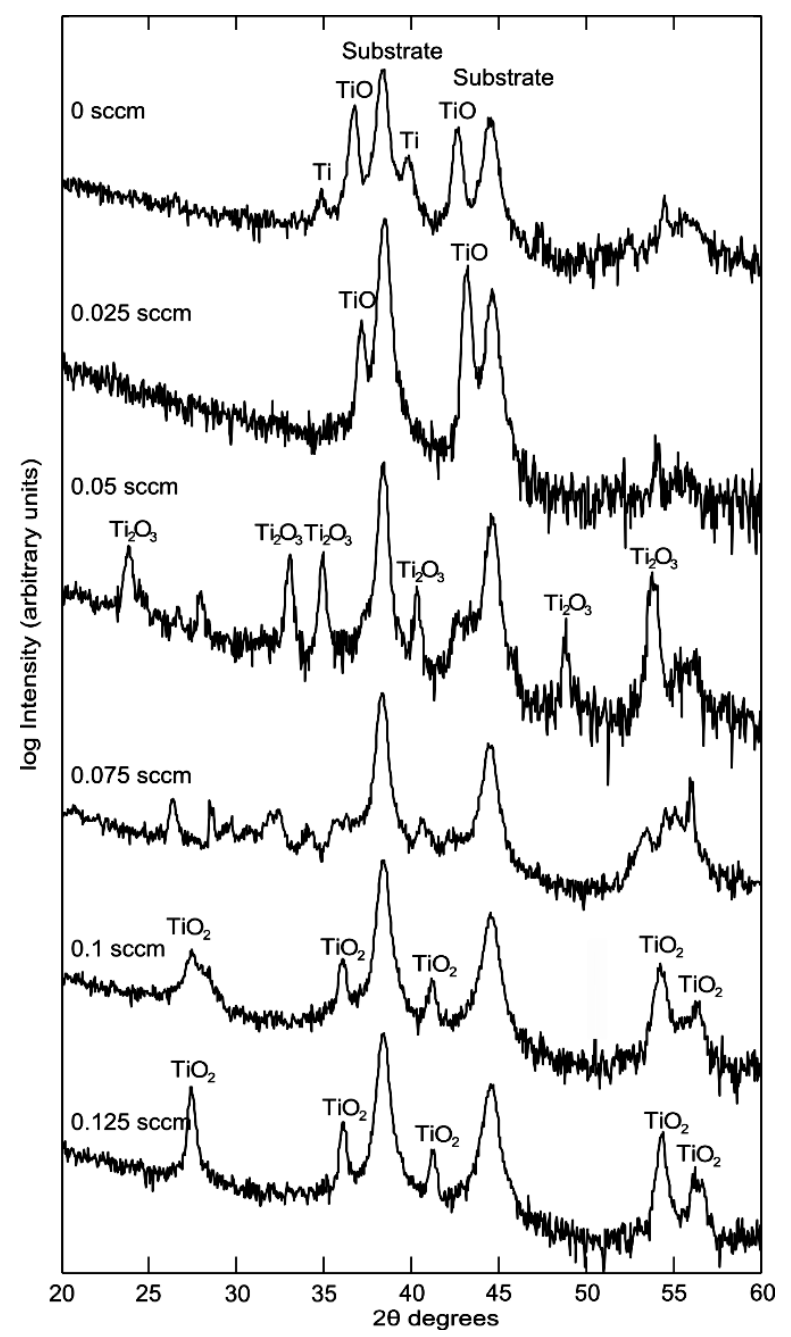

Figure 7: X-ray diffraction patterns on nanoparticles synthesized at different oxygen gas flows. A higher oxygen flow leads to higher oxygen containing phases 
At $0 \mathrm{sccm}$, no oxygen was injected and the oxidation occurred from the residual gas in the vacuum system. Peaks of $\mathrm{Ti}$ and $\mathrm{TiO}$ was found, however it should be pointed out again that these peaks were not reproducible. When $0.025 \mathrm{sccm}$ oxygen was introduced to the process, only $\mathrm{TiO}$ phases were found and at $0.05 \mathrm{sccm} \mathrm{Ti}_{2} \mathrm{O}_{3}$ was the dominating phase. At $0.075 \mathrm{sccm}$, no peaks could be identified and it was probably a mixture of some intermediate phases. When the flow reached $0.1 \mathrm{sccm}$, and above, $\mathrm{TiO}_{2}$ nanoparticles in the rutile phase was found. This result demonstrates the possibility to control the oxygen content of the nanoparticles. This is made possible because the cathode could stay in the transition mode without drifting to the poisoned mode.

The titanium dioxide nanoparticles in the rutile phase were single crystalline. Some particles with twin planes or stacking faults could be found. The particles were fully oxidized all the way through without showing any signs of a less oxidized core [44].

For the titanium particles, there were no significant changes in the particle morphology when their oxygen content increased. This observation is however not universally true, since nanoparticles synthesized with a molybdenum cathode had a profound morphological change. It should be noted that these experiments with molybdenum were run with different discharge parameters $(750 \mathrm{~Hz}, 20 \mathrm{~W})$ and argon gas flows $(5 \mathrm{sccm})$. Here, the particles transitioned from cubic to cauliflower shaped when an oxygen flow of 0.025 was introduced.

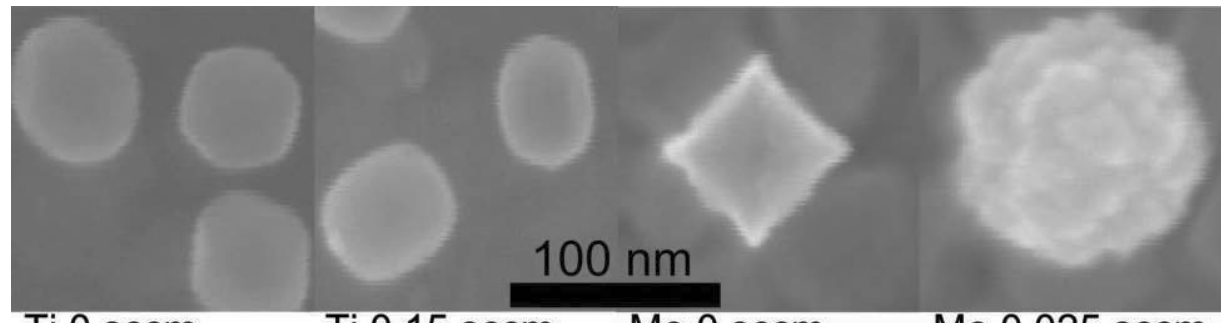

Ti $0 \mathrm{sccm}$ Ti $0.15 \mathrm{sccm}$ Mo $0 \mathrm{sccm}$ Mo $0.025 \mathrm{sccm}$ Figure 8: Nanoparticle change in morphology when oxygen was introduced to the process. No significant change could be seen for the titanium particles. The molybdenum particles transitioned from cubic to a cauliflower morphology. 
This different behaviour between the two materials can be seen in Figure. 8 . The only change in the titanium particles that was readily observed was that the perceived hexagonal shaped particles from the 2 dimensional images disappeared when oxygen was introduced and instead, some particles got a more oval shape. In the case of molybdenum, there was a clear and reproducible transition from cubic to cauliflower shaped particles.

\subsubsection{Oxygens influence on the particle size}

No size variation of the nanoparticles with different oxygen gas flows could be found when particles where synthesized with the titanium hollow cathode. This result is different from those in the literature where titanium oxide nanoparticles were synthesized in a cluster sources. When nanoparticles with sizes of less than $10 \mathrm{~nm}$ were synthesized, an exponential increase of the nanoparticle size with reactive gas flow was found [45]. However, a size decrease has also been found by another group [46]. When a molybdenum cathode was used in the experimental setup described in this thesis, there was a size increase when oxygen was added to the process. So it should not be ruled out that oxygen can have a size increasing effect for different materials or different parameters. No simple explanation and general trend can be made, and the resulting particle size is probably a complicated interplay between particle nucleation and cathode poisoning, which could either increase or decrease the size depending on the experimental setup and the parameters used.

\subsection{Size control}

The ability to control the size of the nanoparticles is one of the most important factors that determines the properties the particles exhibit. It has previously been shown that for copper nanoparticles, the pulse parameters such as frequency can be used to change the nanoparticle size distribution [8]. This approach was also tested for a titanium hollow cathode, but the results were inconclusive. One major reason that these experiments did not work for titanium was that if the discharge parameters were altered so that the amount of sputtered titanium decreased, the cathode went in to the poisoned mode. Because of this, 
other means of size control had to be investigated for titanium nanoparticles.

Size control by changing electrical potentials around the growth zone will first be presented, then a discussion about the influence of pressure and gas flow on the nanoparticle size will be made.

\subsubsection{Size control by changing the mesh bias}

A compilation of nanoparticle size distributions for different mesh biases can be seen in Figure. 9. The open markers represent particles produced at an oxygen gas flow of $0.05 \mathrm{sccm}$. The filled markers represent particles produced at an oxygen flow of $0.15 \mathrm{sccm}$. No trend in the size variation with increasing oxygen gas flow can be seen. There are some size fluctuations between experiments on the same parameters and the fluctuations when oxygen is introduced is in the same order.

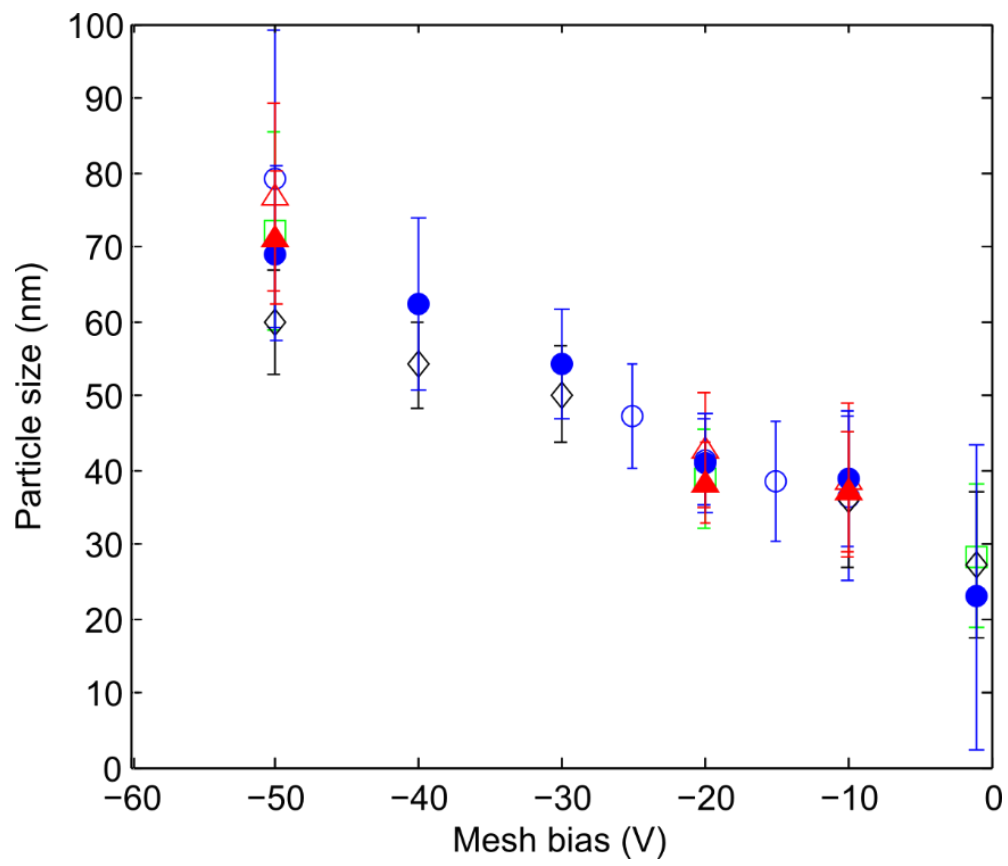

Figure 9: Nanoparticle size as a function of mesh bias for an oxygen flow of 0.05 sccm (open markers) and 0.15 sccm (filled markers). No significant size change can be seen between the two flows. The nanoparticle size decreases with decreasing negative bias. 
There is a clear trend that the size decreases linearly when the negative mesh bias voltage is decreased. The mesh was connected to an active clamp circuit, which allowed current to flow through it if the potential between the mesh and cathode became higher than the potential between cathode and anode. This current was measured and plotted in Figure.10. It can be seen that at $-50 \mathrm{~V}$, all the current passes through the anode. No current is passed through the mesh until the negative mesh bias is reduced to $-20 \mathrm{~V}$. The current then steadily increases with decreasing negative mesh bias.
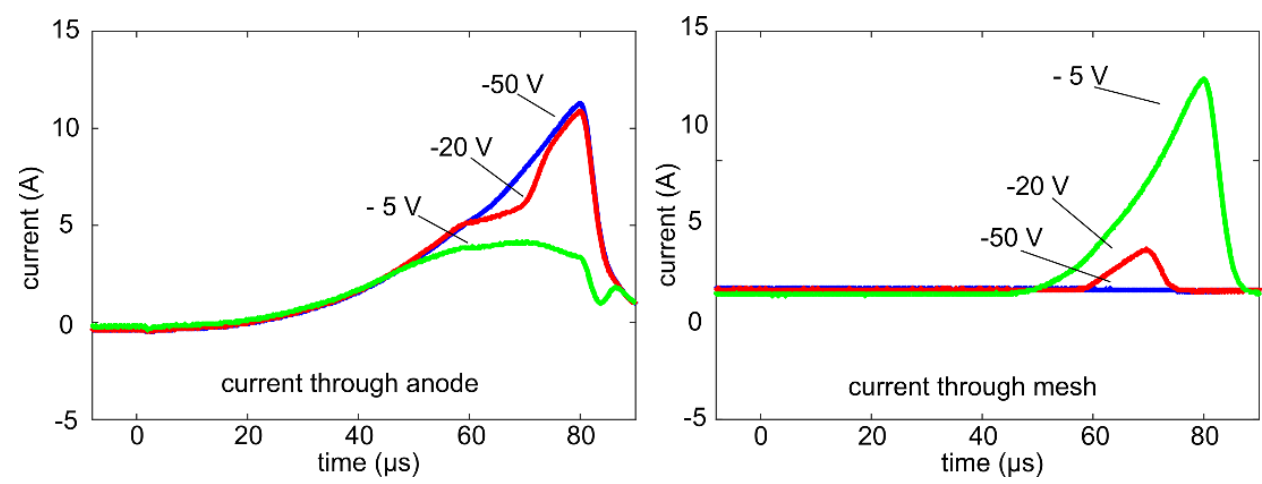

Figure 10 Current through the anode and current through the mesh during the pulse for different mesh biases.

This deflection of the current away from the anode ring and to the mesh also deflects the current away from the zone where the nanoparticles grow. This should decrease the electron temperature, which would lead to a lower ion current which is the main source of growth material to the particles. However, this cannot be the only explanation to this observation. The reason for is that a size decrease is observed between -50 and $-20 \mathrm{~V}$ (Figure. 9), when all of the current still passes through the anode. The real explanation behind how the mesh bias influences the particle size is unknown. By monitoring the partial pressure of oxygen in the process when the mesh bias was changed, it could be seen that this partial pressure was constant. This means that the amount of sputtered titanium that reacted with this oxygen was independent of the mesh bias. The resulting benefit of this is that the particle size can be controlled without influencing the oxygen content of the particle. 


\subsubsection{Size control by pressure and gas flow}

The argon gas pressure and flow was also found to be parameters that influenced the particle size. The following experimental results were obtained with the growth tube. First the influence of the pressure will be presented. Then experiments where the gas flow was varied followed by a simple theoretical model of the observed trends will be discussed.

In Figure. 11, the particles average size and size distributions as a function of pressure is presented for different gas flows. As discussed earlier, at low pressures and high gas flows, oxygen was needed to be added to synthesize particles. This has been marked with a black square behind the markings in the figure. It can be seen that the particle size increases with increasing pressure for all gas flows tested.

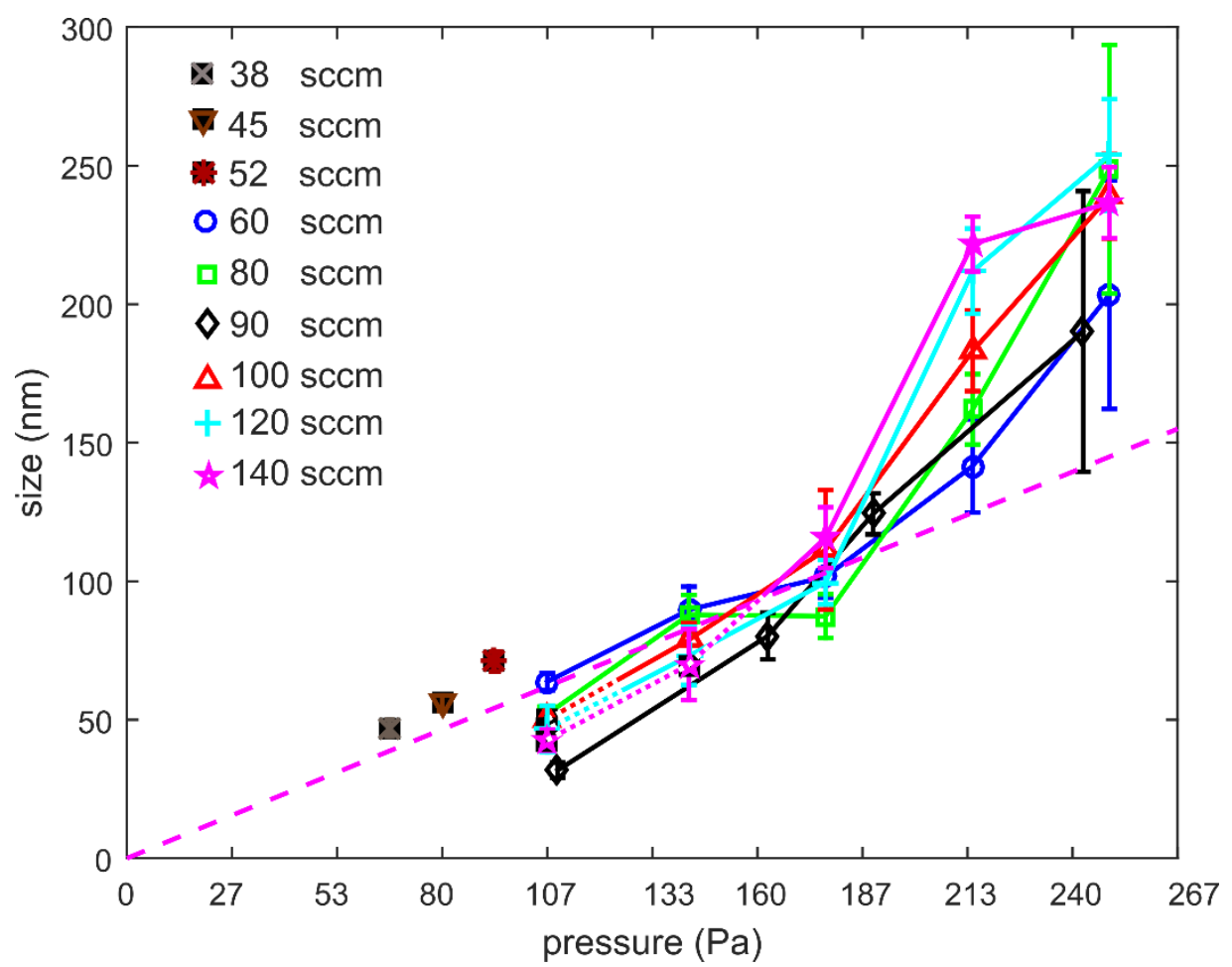

Figure 11: Particle size as a function of pressure for different argon gas flows. The black squares behind the Markings and dotted lines represent regions where oxygen had to be added. The dashed pink line is to show the deviation from a linear trend at $177 \mathrm{~Pa}$. 
This size increase is after $177 \mathrm{~Pa}$ faster than linear as can be seen from the deviation from the pink dashed line. The size distributions also get broader at higher pressures. The size control that is possible by changing the pressure has a much larger range than the mesh bias, however, this pressure increase also increases the oxygen content of the particles. The underlying reason for this increase in oxygen content is believed to be due to a lower pumping speed of reactive gases out of the growth tube at higher pressures. It should also be pointed out that the cathode discharge voltage increases when the pressure is increased which might be an indication of increased cathode oxidation at higher pressures.

The morphology of the particles also changed when the pressure was increased. In Figure. 12 nanoparticles synthesized at a gas flow of 90 sccm at different pressures can be seen. 


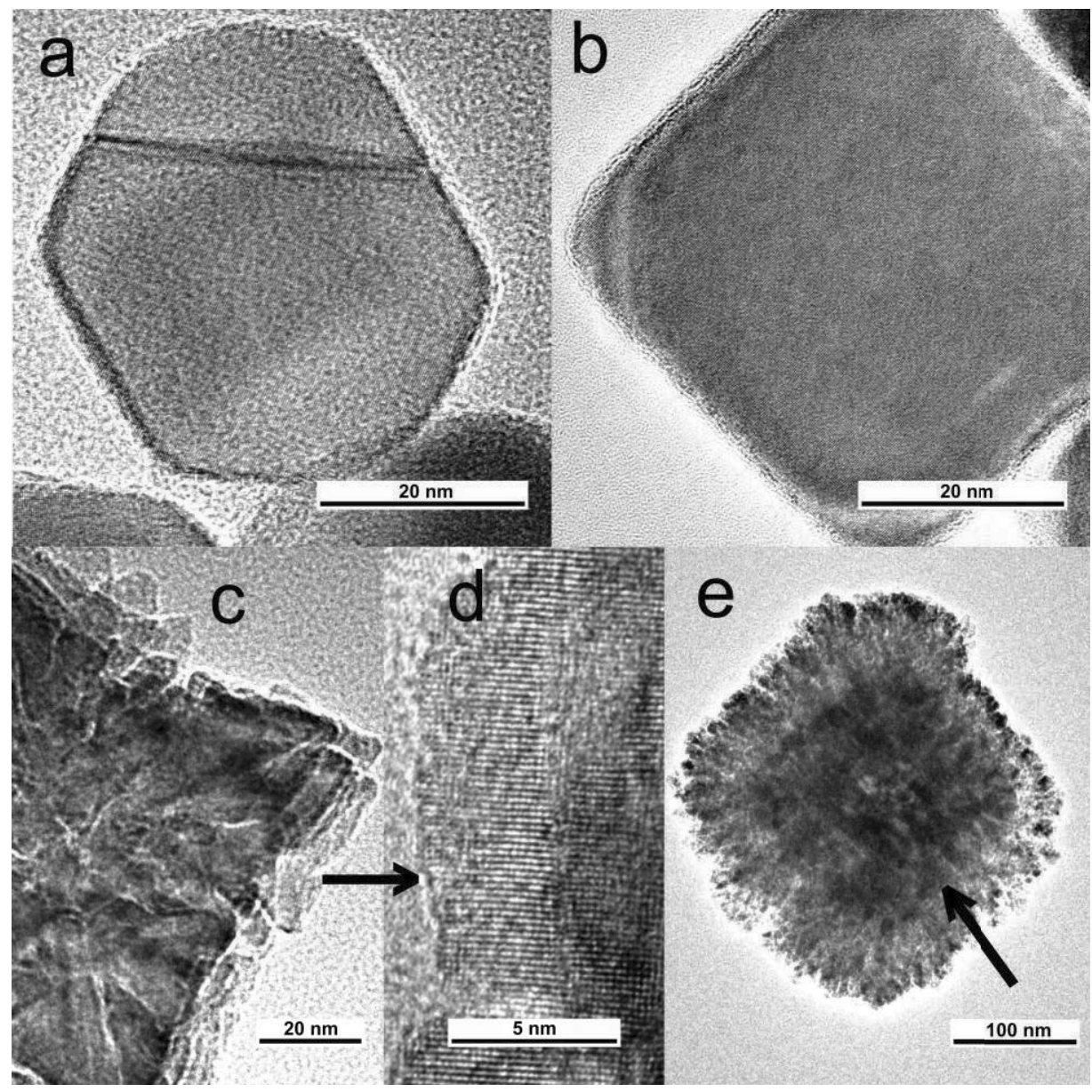

Figure 12: TEM images of particles synthesized at a pressure of 109 Pa (a) 163Pa (b) $189 \mathrm{~Pa}$ (c), (d) and $243 \mathrm{~Pa}$ (e). A close up of the continued single crystal domain at the shell (d). Arrow in (e) shows the cubic core.

The particles start of as faceted crystals where most of them show multiple domains (a). As the pressure is increased, the particles obtain a cubic shape and the whole particle is a single crystalline domain (b). At the pressure of $189 \mathrm{~Pa}$, the particles obtain a faceted shape (c), however they are still single crystalline, as can be seen by the continued crystal domain in (d). When the pressure is increased to $243 \mathrm{~Pa}$, a new morphology emerges (e). This morphology has a single crystalline cubic core (arrow) and a poly crystalline shell and the outer shape of the particle is often referred to as a cauliflower shape. 
This transition from cubic to cauliflower shaped particles with increasing pressure is very similar to the transition of cubic molybdenum particles to cauliflower shape (Figure. 8) when oxygen was introduced to the process. From the literature of thin film growth of molybdenum, it has been observed that columnar grown thin films get smaller columnar grain widths when the oxygen content of the sputtering process is increased. Since this grain width depends on the migration range of the adatoms on the surface, it was argued that since molybdenum has a high affinity to oxygen, the adatom mobility is suppressed by oxidation [47]. This can very well be the case for why the molybdenum particles transition from a cubic to a cauliflower morphology. A low adatom mobility will lead to that the adatoms bind to less preferential sites, which promotes poly crystallinity and thus a cauliflower shaped particle. Similarly in the case for titanium when the pressure is increased, the cooling rate of the particle will increase due to more frequent collisions with argon atoms. A lower temperature will decrease the adatom mobility [48] and thus promote poly crystallinity. In addition to this the deposition rate of titanium on the particle surface could increase. This would promote a more poly crystalline structure, since the adatoms would not have time to find their lowest energy state before getting covered by new atoms [18].

The influence of the inert gas flow rate on the particles will now be presented. The particle size as a function of gas flow for different pressures can be seen in Figure. 13. It was found that the particle size decreased with increasing gas flow at pressures below $143 \mathrm{~Pa}$. Surprisingly at $215 \mathrm{~Pa}$ a particle size increase with increasing gas flow was found. At the pressure of 177 and $249 \mathrm{~Pa}$ no clear trend could be found. 


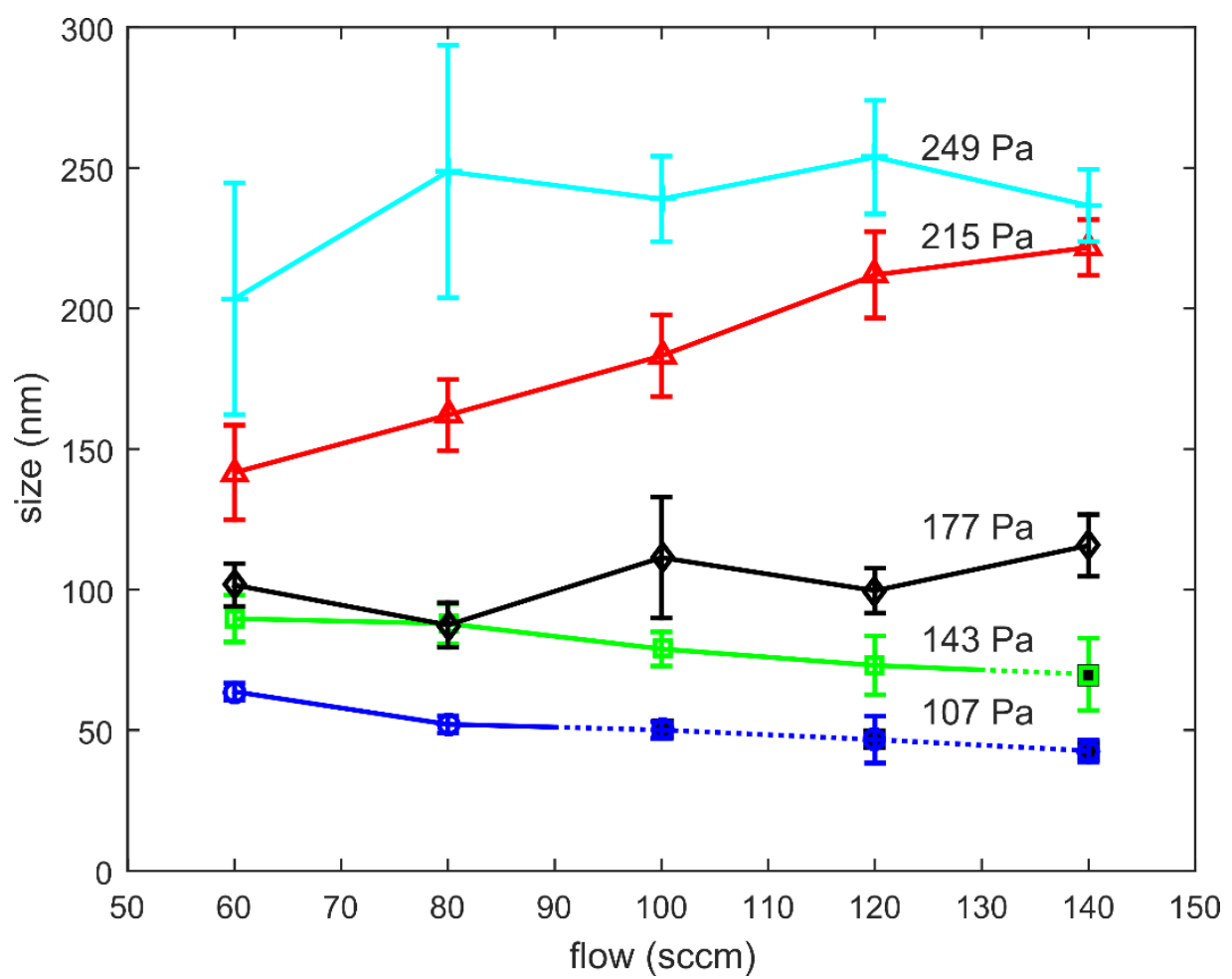

Figure 13: Particle size as function of gas flow for different pressures. The black squares behind the Markings and dotted lines represent regions where oxygen had to be added. At $107 \mathrm{~Pa}$ and $143 \mathrm{~Pa}$, the particle size decreases with increasing flow. At $177 \mathrm{~Pa}$, there is no clear trend in the size as a function of pressure. At $215 \mathrm{~Pa}$ there is a clear size increase with increasing gas flow.

In the literature, when a size decrease is found with an increasing inert gas flow in cluster sources, it is often argued to be due to a shorter residence time of the particles in the growth zone [49]. The same thing can be argued here as well at the lower pressures. Due to the design of cluster sources, the pressure is often set by the gas flow, and thus when the flow is increased, so is the pressure in the aggregation zone [42]. Thus the results in the literature cannot be directly compared to the results found here. The observed size increase could be due to a more efficient extraction of sputtered material from the hollow cathode at the higher pressure [50]. 
A simple model was proposed to explain the observed growth behavior of the particles. The assumptions made and the reasoning behind them can be found in the discussion section of paper 2. In short it is assumed that the particle growth rate can be explained by

$$
r_{N P}=\left\langle d r_{N P} / d t\right\rangle \cdot t_{r e s}
$$

Where $r_{N P}$ is the particle size, $d r_{N P} / d t$ is the particle growth rate and $t_{\text {res }}$ is the residence time of the particle in the growth zone. As was found in Figure. 11, the growth rate does not increase linearly with pressure but rather exponential. Thus both the growth rate and the residence time should increase linearly with pressure, in order to give an exponential increase. Why the growth rate would increase with pressure can be understood by that the diffusion rate of titanium ions from the cathode to the wall decreases with increasing pressure. Thus a higher density of titanium would be available at higher pressures which increases the growth rate. To explain why the residence time would increase with pressure one has to first mention that the sputtered material from the hollow cathode gets ejected by a puff, that can reach velocities of $190 \mathrm{~m} / \mathrm{s}$ [16]. The ejected material/particles will then be slowed down by the gas in the growth tube, which has a much lower velocity. The friction between the gas and the particles will increase with pressure, and thus the particles will stay longer in the growth tube for higher pressures. 



\section{Concluding remarks}

Nanoparticles of titanium have been synthesized in a plasma generated by a pulsed hollow cathode sputtering process. The reactive gas partial pressure originating from oxygen or water vapor was found to be an important parameter. If the reactive gas was too low, no nucleation occurred. If the reactive gas was too high, the cathode oxidized which reduced the yield of particles and made the process unstable. In order to synthesize particles, this reactive gas had to be in an intermediate range. By varying the oxygen partial pressure inside this range, the oxygen stoichiometry of the particles could be controlled. This was made possible since the use of a hollow cathode with an inert gas flow through it reduced the oxidation of the cathode surface which stabilized the sputtering process in the transition mode. To further suppress the inflow of reactive from the vacuum system to the growing particles, a tube was put around the growth zone. This allowed for deposition conditions that were cleaner and more stable.

It was possible to control the nanoparticle size without influencing their oxygen content by changing electrical potentials around the growth zone. The pressure was found to change the morphology of the particles which was attributed to a lower adatom mobility on the particle surface at higher pressures. A pressure increase also led to an exponential increase of the particle size. This could be explained by that both the growth rate and the residence time of the particles in the plasma increased with increasing pressure. The growth rate increase is due to a higher plasma density due to a lower ambipolar diffusion rate. The longer residence time can be attributed to a higher friction between the particles and the surrounding gas at the higher pressure. 



\section{Current research and future plans}

Although the reproducibility of the nanoparticle synthesis process increased with the growth tube attached, the deposition rate is still not controllable. This is because the process consumes water vapor which slowly gets depleted that leads to a decreased deposition rate. In addition to this, particles with an oxygen content of less than $20 \%$ cannot be synthesized. The underlying reason why pressure was investigated was to try to find a parameter which made clean particles that were not contaminated with oxygen. The idea was to try to enter a homogenous nucleation regime, and thus not be dependent on the fluctuating partial pressure of water in order to get nucleation. This was a real problem when a side project needed InAlN particles, but only InAlON particles with uncontrollable deposition rate and oxygen content could be produced. Thus cleaner and more stable parameters need to be investigated in the future.

Since higher pressures increased the oxygen content of the particles it would in the future be interesting to synthesize particles at cleaner conditions in higher pressures. This could be done by re designing the growth tube to be allowed to be baked out before depositions. In combination with the higher pressure, the use of pulsing parameters that gives a higher supersaturation of the titanium vapor could be used. In addition to this, applying magnetic fields around the growth zone could also be tested in order to confine the plasma and thus increase the super saturation.

If this is shown to not work, and oxygen is still found to be necessary to get a decent production of particles, a method for intentionally injecting a low amount of oxygen that is high enough to allow for nucleation, but low enough to not severely contaminate the particles or the cathode has to be invented. Hydrogen has been used in order to reduce oxygen from thin films, so a combination of hydrogen and oxygen might be a rout to take. 



\section{References}

[1] W.J. Stark, P.R. Stoessel, W. Wohlleben, A. Hafner, Chem Soc Rev Industrial applications of nanoparticles, Chem. Soc. Rev. 44 (2015) 5793-5805.

[2] X. Chen, S.S. Mao, Titanium dioxide nanomaterials: synthesis, properties, modifications, and applications., Chem. Rev. 107 (2007) 2891-959.

[3] P. Spinelli, V.E. Ferry, J. Van De Groep, M. Van Lare, M.A. Verschuuren, R.E.I. Schropp, et al., Plasmonic light trapping in thin-film Si solar cells, J. Opt. 14 (2012) 024002.

[4] A. Ghaderi, E.A. De Mayolo, H.K. Patra, M. Golabi, Keys and regulators of nanoscale theranostics, Adv. Mater. Lett. 6 (2015) 87-98.

[5] D.A. Links, New insight into the soot nanoparticles in a candle flame, Chem. Commun. 47 (2011) 4700-4702.

[6] A. Betke, G. Kickelbick, Bottom-Up, Wet Chemical Technique for the Continuous Synthesis of Inorganic Nanoparticles, Inorganics. 2 (2014) 1-15.

[7] F. Kruis, H. Fissan, a Peled, Synthesis of nanoparticles in the gas phase for electronic, optical and magnetic applications - a review, J. Aerosol Sci. 29 (1998) 511-535.

[8] I. Pilch, D. Söderström, N. Brenning, U. Helmersson, Sizecontrolled growth of nanoparticles in a highly ionized pulsed plasma, Appl. Phys. Lett. 102 (2013) 033108.

[9] I. Pilch, D. Söderström, M.I. Hasan, U. Helmersson, N. Brenning, Fast growth of nanoparticles in a hollow cathode plasma through orbit motion limited ion collection, Appl. Phys. Lett. 103 (2013) 193108.

[10] John F. O'Hanlon, A User's Guide to Vacuum Technology, 3rd Edition, Wiley-Interscience, 2003.

[11] A. Anders, J. Andersson, A. Ehiasarian, High power impulse magnetron sputtering: Current-voltage-time characteristics indicate the onset of sustained self-sputtering, J. Appl. Phys. 102 (2007) 113303.

[12] W. He, W. Lv, J. Dickerson, Gas Transport in Solid Oxide Fuel Cells, Springer, 2014.

[13] R.C. Krutenat, C. Panzera, Low - Energy Ar + Sputtering Yields 
of Solid and Liquid Tin Low-energy sputtering yields of tungsten and tantalum Low - Energy Sputtering Yields of Ge Single Crystals as a Function of Temperature, J. Appl. Phys. 41 (1970) 4953.

[14] A. Data, Y. Yamamura, H. Tawara, Energy Dependence of Ioninduced Sputtering Yields From Monatomic Solids at Normal Incidence, At. DATA Nucl. DATA TABLES. 62 (1996) 149-253.

[15] C. Steinbriichel, A Simple Formula for Low-Energy Sputtering Yields, Appl. Phys. A. 36 (1985) 37-42.

[16] M.I. Hasan, I. Pilch, D. Söderström, D. Lundin, U. Helmersson, N. Brenning, Modeling the extraction of sputtered metal from high power impulse hollow cathode discharges, Plasma Sources Sci. Technol. 22 (2013) 035006.

[17] H. Haberland, M. Karrais, M. Mall, Y. Thurner, Thin films from energetic cluster impact : A feasibility study, J. Vac. Sci. Technol. A. 10 (1992) 3266-3271.

[18] M. Ohring, The Material Science of Thin Films, Academic Press Limited, 1992.

[19] Diederik Depla, Magnetrons, reactive gases and sputtering, 2013.

[20] D. Söderström, Modelling and Applications of the Hollow Cathode Plasma, 2008.

[21] W.D. Sproul, D.J. Christie, D.C. Carter, Control of reactive sputtering processes, Thin Solid Films. 491 (2005) 1-17.

[22] T. Nyberg, S. Berg, U. Helmersson, K. Hartig, Eliminating the hysteresis effect for reactive sputtering processes, Appl. Phys. Lett. 86 (2005) 164106.

[23] M. Aiempanakit, T. Kubart, P. Larsson, K. Sarakinos, J. Jensen, U. Helmersson, Hysteresis and process stability in reactive high power impulse magnetron sputtering of metal oxides, Thin Solid Films. 519 (2011) 7779-7784.

[24] E. Wallin, U. Helmersson, Hysteresis-free reactive high power impulse magnetron sputtering, Thin Solid Films. 516 (2008) 63986401.

[25] T. Kalber, Gas flow sputtering of oxide coatings: practical aspects of the process, Surf. Coatings Technol. 86-87 (1996) 218-224.

[26] M. Aiempanakit, A. Aijaz, D. Lundin, U. Helmersson, T. Kubart, Understanding the discharge current behavior in reactive high power impulse magnetron sputtering of oxides, J. Appl. Phys. 113 (2013) 133302. 
[27] A. Bouchoule, Dusty Plasmas Physics, Chemistry and Technological Impacts in Plasma Processing, Wiley, 1999.

[28] A. Piel, Plasma Physics. An introduction to Laboratory, Space and Fusion Plasmas, Springer, 2010.

[29] A. Gallagher, Model of particle growth in silane discharges, Phys. Rev. E. 62 (2000) 2690-2706.

[30] L. Ravi, S.L. Girshick, Coagulation of nanoparticles in a plasma, Phys. Rev. E. 79 (2009) 026408.

[31] A. Piel, Plasma Physics. An introduction to laboratory, Space and Fusion Plasmas, Springer, 2010.

[32] K. Wegner, P. Piseri, H.V. Tafreshi, P. Milani, Cluster beam deposition : a tool for nanoscale science and technology, J. Phys. D Appl. Phys. 39 (2006) R439-R459.

[33] T. Peter, O. Polonskyi, B. Gojdka, A. Mohammad Ahadi, T. Strunskus, V. Zaporojtchenko, et al., Influence of reactive gas admixture on transition metal cluster nucleation in a gas aggregation cluster source, J. Appl. Phys. 112 (2012) 114321.

[34] A.M. Ahadi, V. Zaporojtchenko, T. Peter, O. Polonskyi, T. Strunskus, F. Faupel, Role of oxygen admixture in stabilizing TiO $\mathrm{x}$ nanoparticle deposition from a gas aggregation source, $\mathrm{J}$. Nanoparticle Res. 15 (2013) 2125.

[35] P. V. Kashtanov, B.M. Smirnov, R. Hippler, Efficiency of cluster generation in a magnetron discharge, EPL (Europhysics Lett. 91 (2010) 63001.

[36] O. Polonskyi, T. Peter, A. Mohammad Ahadi, A. Hinz, T. Strunskus, V. Zaporojtchenko, et al., Huge increase in gas phase nanoparticle generation by pulsed direct current sputtering in a reactive gas admixture, Appl. Phys. Lett. 103 (2013) 033118.

[37] T. Acsente, R.F. Negrea, L.C. Nistor, C. Logofatu, E. Matei, C. Grisolia, et al., Synthesis of flower-like tungsten nanoparticles by magnetron sputtering combined with gas aggregation, Eur. Phys. J. D. 69 (2015) 161.

[38] A. Marek, J. Valter, S. Kadlec, J. Vyskočil, Gas aggregation nanocluster source - Reactive sputter deposition of copper and titanium nanoclusters, Surf. Coatings Technol. 205 (2011) S573S576.

[39] C. Clavero, J.L. Slack, A. Anders, Size and compositioncontrolled fabrication of thermochromic metal oxide nanocrystals, J. Phys. D. Appl. Phys. 46 (2013) 362001. 
[40] V.M. Smirnov, Processes in expanding and condensing gases, Physics-Uspekhi. 37 (1994) 646.

[41] R.D. Boyd, I. Pilch, M. Garbrecht, M. Halvarsson, U. Helmersson, Double oxide shell layer formed on a metal nanoparticle as revealed by aberration corrected ( scanning) transmission electron microscopy, Mater. Res. Express. 1 (2014) 025016.

[42] M. Drábik, A. Choukourov, A. Artemenko, J. Kousal, O. Polonskyi, P. Solař, et al., Morphology of Titanium Nanocluster Films Prepared by Gas Aggregation Cluster Source, Plasma Process. Polym. 8 (2011) 640-650.

[43] N. Tanaka, D. Peng, K. Sumiyama, T. Hihara, Face-Centered Cubic Ti Cluster Assemblies Prepared by Plasma-GasCondensation, Mater. Trans. 49 (2008) 522-526.

[44] R.D. Boyd, R. Gunnarsson, I. Pilch, U. Helmersson, Characterisation of Nanoparticle Structure by High Resolution Electron Microscopy ., J. Phys. Conf. Ser. 522 (2014) 012065.

[45] A.M. Ahadi, O. Polonskyi, U. Schürmann, Stable production of $\mathrm{TiO} \times$ nanoparticles with narrow size distribution by reactive pulsed dc magnetron sputtering, J. Phys. D. Appl. Phys. 48 (2015) 35501.

[46] J. Hanu, A. Choukourov, I. Melnichuk, H. Biederman, A. Serov, D. Slavínsk, Preparation of metal oxide nanoparticles by gas aggregation cluster source, Vacuum. 120 (2015) 162-169.

[47] H. Search, C. Journals, A. Contact, M. Iopscience, I.P. Address, Effects of Oxygen on the Properties of Sputtered Molybdenum Thin Films, Jpn. J. Appl. Phys. 30 (1991) 2069-2073.

[48] P. Patsalas, C. Charitidis, S. Logothetidis, The effect of substrate temperature and biasing on the mechanical properties and structure of sputtered titanium nitride thin films, Surf. Coatings Technol. 125 (2000) 335-340.

[49] S. Srivastava, J.P. Thomas, A. Rahman, M. Abd-ellah, M. Mohapatra, D. Pradhan, et al., Size-Selected TiO 2 Nanocluster Catalysts for Effi cient Photoelectrochemical Water Splitting, ACS Nano. 8 (2014) 11891-11898.

[50] M. Maicu, R. Schmittgens, D. Hecker, D. Glöß, P. Frach, G. Gerlach, et al., Synthesis and deposition of metal nanoparticles by gas condensation process, J. Vac. Sci. Technol. A. 32 (2014) 02B113. 


\section{Papers}

The articles associated with this thesis have been removed for copyright reasons. For more details about these see:

http://urn.kb.se/resolve?urn=urn:nbn:se:liu:diva-128622 